\title{
Immunomodulatory role of nonneuronal cholinergic signaling in myocardial injury
}

\author{
Cibele Rocha-Resende, Carla Weinheimer, Geetika Bajpai, Luigi Adamo, Scot J. Matkovich, \\ Joel Schilling, Philip M. Barger, Kory J. Lavine, and Douglas L. Mann \\ Cardiovascular Division, Department of Medicine, Washington University School of Medicine, St. Louis, Missouri, USA.
}

\begin{abstract}
Whereas prior studies have demonstrated an important immunomodulatory role for the neuronal cholinergic system in the heart, the role of the nonneuronal cholinergic system is not well understood. To address the immunomodulatory role of the nonneuronal cholinergic system in the heart, we used a previously validated diphtheria toxin-induced (DT-induced) cardiomyocyte ablation model (Rosa26-DTMic2v-Cre mice). DT-injected Rosa26-DT ${ }^{\text {Mlc2v-Cre }}$ mice were treated with diluent or pyridostigmine bromide (PYR), a reversible cholinesterase inhibitor. PYR treatment resulted in increased survival and decreased numbers of $\mathrm{MHC}-\mathrm{II}^{\circ} \mathrm{CCR2}{ }^{+}$macrophages in DTinjected Rosa26-DT ${ }^{\text {Mic2v-Cre }}$ mice compared with diluent-treated Rosa26-DT ${ }^{\text {Mic2v-Cre }}$ mice. Importantly, the expression of CCL2/7 mRNA and protein was reduced in the hearts of PYR-treated mice.

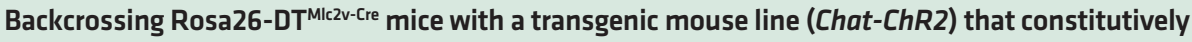
overexpresses the vesicular acetylcholine transporter (VAChT) resulted in decreased expression of $\mathrm{Ccl} 2 / 7$ mRNA and decreased numbers of $\mathrm{CD68}^{+}$cells in DT-injured Rosa26-DT ${ }^{\text {Mic2v-Cre }} /$ Chat-ChR2 mouse hearts, consistent with the pharmacologic studies with PYR. In vitro studies with cultures of LPS-stimulated peritoneal macrophages revealed a concentration-dependent reduction in CCL2 secretion following stimulation with acetylcholine, nicotine, and muscarine. To our knowledge, these findings reveal a previously unappreciated immunomodulatory role for the nonneuronal cholinergic system in regulating homeostatic responses in the heart following tissue injury.
\end{abstract}

Conflict of interest: The authors have declared that no conflict of interest exists.

Copyright: () 2019, American Society for Clinical Investigation.

Submitted: March 20, 2019

Accepted: May 30, 2019

Published: July 25, 2019.

Reference information: /Cl Insight. 2019;4(14):e128961. https://doi. org/10.1172/jici.nsight.128961.

\section{Introduction}

The nervous system and the immune system, which coevolved under selective pressures imposed by infection and tissue injury, are essential for coordinating host responses to environmental danger (1). A classic example of the reciprocal interaction of the central nervous system and the immune system is the febrile response orchestrated by activation of the immune system in response to an infection (2). The nervous system and the immune system are integrally linked through multiple anatomic connections, the most notable of which is the innervation of lymphoid tissue (spleen, bone marrow, lymph nodes, and thymus) by the autonomic nervous system. The parasympathetic nervous system innervates lymphoid tissue via the neurotransmitter acetylcholine (ACh), and the sympathetic nervous system innervates lymphoid tissue via the neurotransmitter norepinephrine (3). The role of the parasympathetic nervous system in modulating inflammation has been recently established in a series of elegant studies that have identified the cholinergic antiinflammatory pathway, wherein peripheral afferent vagal nerves transmit danger signals from the periphery to integrative regions within the brain stem that reciprocally activate vagal efferent fibers that terminate in the spleen and other lymphoid tissues. Stimulation of vagal efferent in the spleen results in the local release of ACh that inhibits the release of proinflammatory cytokines by macrophages (4-6).

$\mathrm{ACh}$ is synthesized in the cytoplasm by choline acetyltransferase (ChAT), transported into synaptic vesicles by vesicular ACh transporter (VAChT), and then stored in the cytosol until it is released into the extracellular space, where it signals through activation of nicotinic (nAChR) or muscarinic (mAChR) receptors. ACh is degraded in the extracellular space by cholinesterases, which are expressed ubiquitously in tissues (7). Although ACh is widely recognized as a classic neurotransmitter, it has also become apparent that $\mathrm{ACh}$ is also secreted by nonneuronal cell types, where it is involved in a variety of homeostatic cellular functions (8). Germane to this discussion, recent studies have shown that ACh is synthesized by cardiomyocytes. ACh that is secreted by cardiomyocytes is cytoprotective (9-12), although the mechanism(s) for this cytoprotection is not well understood. Noting that neuronal $\mathrm{ACh}$ activated by the vagus nerve inhibits 
the release of proinflammatory cytokines during sepsis (4) and that the nonneuronal cholinergic machinery is present in cardiomyocytes, immune cells (13), and in endothelial cells (14), we asked whether the cytoprotective effects of nonneuronal ACh in the heart were secondary, at least in part, to regulation of the immune system. Here, we show that augmenting nonneuronal cholinergic signaling using either pharmacologic or genetic approaches confers beneficial immunomodulatory effects, by decreasing the expression of CCL2/7 chemokines, as well as decreased influx of proinflammatory CCR2 ${ }^{+}$monocytes to the heart following tissue injury.

\section{Results}

Treatment with pyridostigmine attenuates cardiac injury and cardiac inflammation. To study the role of the nonneuronal ACh system in modulating cardiac injury, we employed a diphtheria toxin-based (DT-based) model system (15), wherein the DT injection selectively ablates cardiomyocytes that have been genetically engineered to express the DT receptor (Rosa26-DT ${ }^{\text {Mlc2v-Cre }}$ mice). The rationale for choosing a genetic model of cardiac injury versus more conventional pathophysiological models of cardiac injury, such as acute coronary artery ligation or ischemia/reperfusion injury was 2-fold: first, thoracotomy results in dysregulation of the autonomic nervous system for up to 7 days after surgery (16), and second, in preliminary control experiments designed to evaluate perioperative responses we observed that thoracotomy provokes a significant inflammatory response in the heart for up to 5 days, in the absence of discrete tissue injury (see Supplemental Figure 1; supplemental material available online with this article; https://doi.org/10.1172/jci.insight.128961DS1).

Rosa26-DT ${ }^{\text {Mlc2v-Cre }}$ mice and littermate control (LM) mice were treated with diluent (PBS) or pyridostigmine bromide (PYR), which was used to inhibit cholinesterase. As shown in Figure 1A, there were no deaths in the diluent- and PYR-treated LM mice. In contrast, there was a striking increase in mortality in DT-injected Rosa26-DT ${ }^{\text {Mlc2v-Cre }}$ mice treated with diluent (Rosa26-DT ${ }^{\text {Mlc2v-Cre }} / \mathrm{PBS}$ ) that began as early as day 2, whereas the onset of mortality in DT-injected PYR-treated mice (Rosa26-DT ${ }^{\text {Mic2v-Cre } / P Y R) ~ w a s ~}$ delayed. Analysis of the Kaplan-Meier survival curves (Figure 1A) revealed that there was a significant $(P$ $=0.01$ ) decrease in mortality in the Rosa26-DT ${ }^{\text {Mlc2v-Cre } / P Y R ~ m i c e ~ w h e n ~ c o m p a r e d ~ w i t h ~ R o s a 26-D T ~}{ }^{\text {Mlc2v-Cre / }}$ PBS mice. To determine whether differences in the extent of tissue injury were responsible for the early increase in mortality in the Rosa26-DT ${ }^{\mathrm{Mlc2v}-\mathrm{Cre}} / \mathrm{PBS}$ mice, we measured the uptake of Evans Blue dye and serum troponin I levels on day 3. Figure 1, B-D, shows that Evans Blue dye uptake and serum troponin I levels were not significantly different $\left(P>0.99\right.$ for both) in the Rosa26-DT ${ }^{\text {Mlc2v-Cre }} / \mathrm{PBS}$ and Rosa26-DTMlc2v-Cre/PYR mice, suggesting that differences in DT-mediated tissue injury were not responsible for the increased mortality in the Rosa26-DT ${ }^{\mathrm{Mlc} 2 \mathrm{v}-\mathrm{Cre}} / \mathrm{PBS}$ mice. In contrast to the findings obtained on day 3, there was a significant $(P=0.01)$ increase in the troponin I levels on day 5 in the Rosa26-DT ${ }^{\text {Mlc2v-Cre }} / \mathrm{PBS}$ mice when compared with the Rosa26-DT ${ }^{\mathrm{Mlc} 2 \mathrm{v}-\mathrm{Cre}} / \mathrm{PYR}$ mice (Figure 1D), consistent with a delayed increase in tissue injury in the Rosa26-DTMlc2v-Cre $/ P B S$ mice.

Given the role of $\mathrm{ACh}$ in modulating the immune system, we next asked whether there were differences in the inflammatory response to tissue injury in the Rosa26-DT ${ }^{\mathrm{Ml} 2 \mathrm{v}-\mathrm{Cre}} / \mathrm{PBS}$ and Rosa26-DT ${ }^{\mathrm{Mlc} 2 \mathrm{~V}-\mathrm{Cr}} /$ PYR mouse hearts. As shown in Figure $1, \mathrm{E}$ and $\mathrm{F}$, there was a striking $(P<0.0001)$ increase in the inflammatory infiltrate in Rosa26-DT ${ }^{\text {Mlc2v-Cre }} / \mathrm{PBS}$ hearts when compared with diluent-treated LMs (LM/PBS). In contrast, the inflammatory score $(17)$ was significantly $(P=0.01)$ decreased in the Rosa26-DT ${ }^{\text {Mlc2v-Cre/ }}$ PYR hearts when compared with Rosa26-DT ${ }^{\mathrm{Mlc2v}-\mathrm{Cre}} / \mathrm{PBS}$ hearts (Figure 1, E and F), although leukocyte infiltration was still significantly $(P=0.03)$ greater than that observed in PYR-treated LMs (LM/PYR).

To begin to explore the mechanisms for the differences in inflammation in the hearts of the Rosa26-DTMlc2v-Cre/PBS and Rosa26-DT ${ }^{\text {Mlc2v-Cre } / P Y R ~ m i c e, ~ w e ~ p e r f o r m e d ~ t r a n s c r i p t i o n a l ~ p r o f i l i n g ~ o n ~ d a y ~} 5$ after DT injection. As shown in Figure 2A, 6149 transcripts were differentially regulated in Rosa26-DT ${ }^{\mathrm{Mlc} 2 \mathrm{-C} \text {-Ce/ }}$ PBS versus LM/PBS hearts, whereas 1721 transcripts were differentially regulated in Rosa26-DT ${ }^{\text {Mlc2v-Cre/ }}$ PYR versus Rosa26-DT ${ }^{\text {MIc2v-Cre }} / \mathrm{PBS}$ hearts. Of the 6149 transcripts that were differentially regulated in the Rosa26-DT ${ }^{\text {Mic2v-Cre } / P B S ~ v e r s u s ~ L M / P B S ~ h e a r t s, ~ t r e a t m e n t ~ w i t h ~ P Y R ~ n o r m a l i z e d ~ t h e ~ e x p r e s s i o n ~ o f ~} 1693$ transcripts, of which 1113 were initially upregulated and 580 were initially downregulated. The principal component analysis plot illustrated in Figure 2B shows that the transcriptional profile of the Rosa26-DTMlc2v-Cre /PYR mice was intermediate between Rosa26-DT ${ }^{\text {Mlc2v-Cre } / P B S ~ h e a r t s ~ a n d ~ t h e ~ L M / P B S ~ a n d ~ L M / ~}$ PYR hearts, suggesting that PYR treatment resulted in partial resolution of the dysregulated transcriptional profile following DT-induced cardiac injury. We next performed a KEGG pathways analysis on the 1693 genes that were normalized (relative fold change $\geq 1.5$, FDR $<0.05$ ) in the PYR-treated Rosa26-DT ${ }^{\mathrm{Mlc} 2 \mathrm{~V}-\mathrm{Cre}}$ 
A

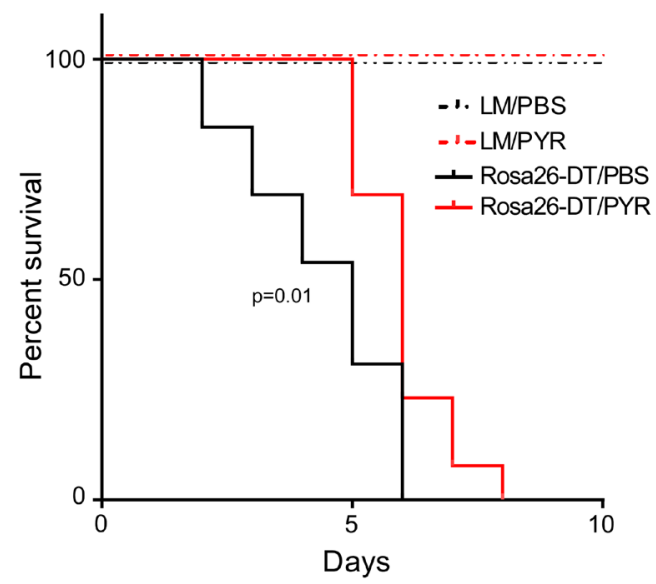

C

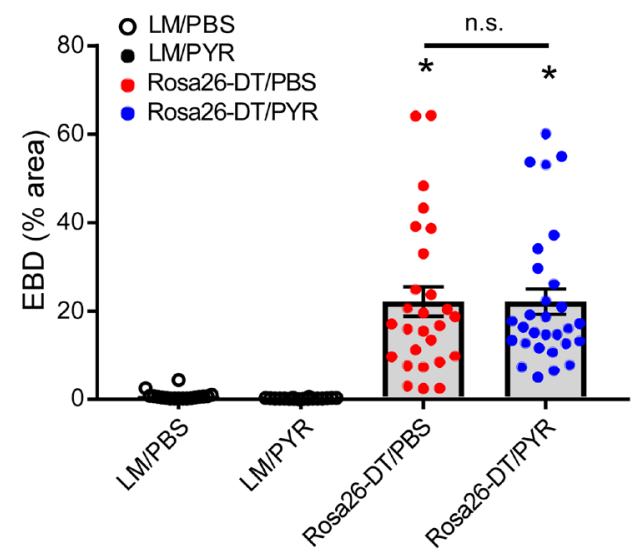

$\mathbf{E}$
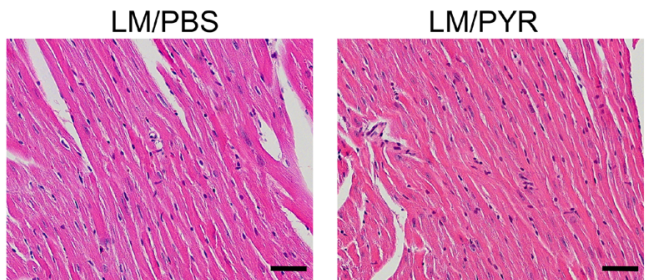

Rosa26-DT/PBS

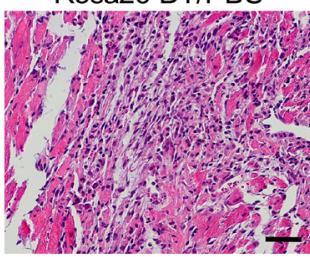

B
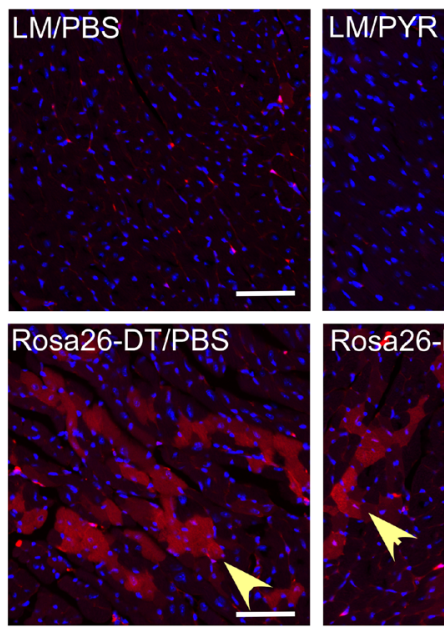

D

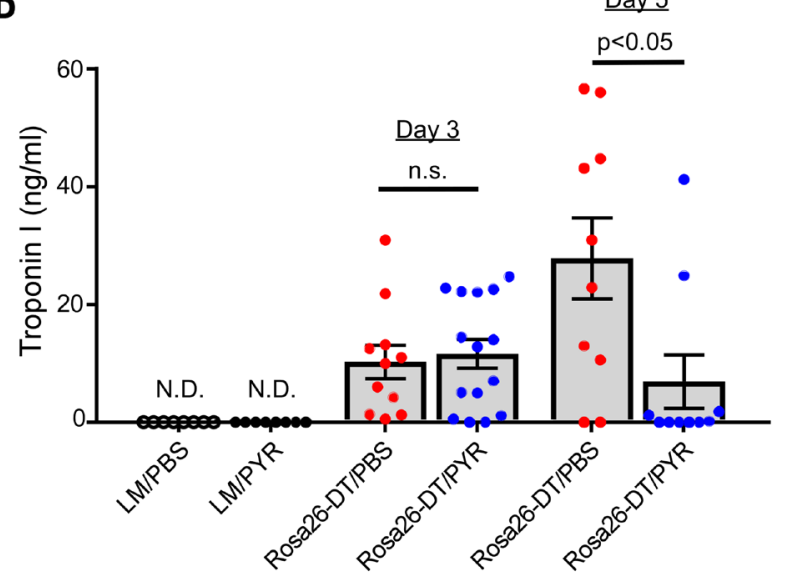

$\mathbf{F}$

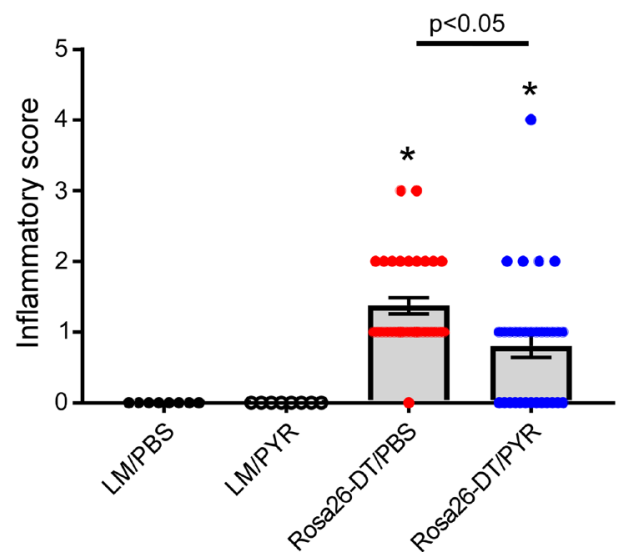

Figure 1. Effect of pyridostigmine treatment on survival, tissue injury, and inflammation following diphtheria toxin-mediated injury. (A) Kaplan-Meier survival curves for diphtheria toxin-injected (DT-injected) Rosa26-DTMicz-cre (Rosa26-DT) and littermate control (LM) mice treated with pyridostigmine (PYR) or diluent (PBS, $n=13$ mice/group). (B) Representative photomicrographs of Evans Blue dye (EBD) uptake by cardiomyocytes (yellow arrowhead) on day 3 after DT injection in LM and Rosa26-DTMic2v-Cre mice treated with PYR or diluent. Scale bar: $50 \mu \mathrm{m}$. (C) Summary of group data for EBD uptake on day 3 after DT injection in LM and Rosa26-DTMicz-Cre mice treated with PYR or diluent ( $n=18-29$ fields obtained from 3 hearts/condition). (D) Serum troponin I $(\mathrm{ng} / \mathrm{mL}$ ) levels on day 3 and day 5 after DT injection in LM and Rosa26-DTMic2v-cre mice treated with PYR or diluent ( $n=8-15$ mice/condition). (E) Representative photomicrographs of hematoxylin and eosin-stained hearts on day 5 after DT injection in LM and Rosa26-DTMic2v-rre mice treated with PYR or diluent. Scale bar: $50 \mu \mathrm{m}$. (F) Group data of the inflammatory score on day 5 after DT injection in LM and Rosa26-DT ${ }^{\text {Miczv-cre }}$ mice treated with PYR or diluent ( $n=$ 31-32 fields obtained from 4 hearts/condition). 0 , no infiltrate; $1+$, infiltrates involving $<25 \%$ myocardium; $2+$, infiltrates involving $25 \%-50 \%$; $3+$, infiltrates involving $50 \%-75 \%$ of the myocardium; and $4+$, infiltrates involving $75 \%-100 \%$ of the myocardium. N.D., not detected. ${ }^{*} P<0.05$ in comparison with LM/PBS and LM/PYR. $P$ values were calculated with Gehan-Breslow-Wilcoxon test for $\mathbf{A}$ and 1-way ANOVA followed by the Tukey post hoc test for $\mathbf{C}$ $\mathbf{D}$, and $\mathbf{F}$. 
A

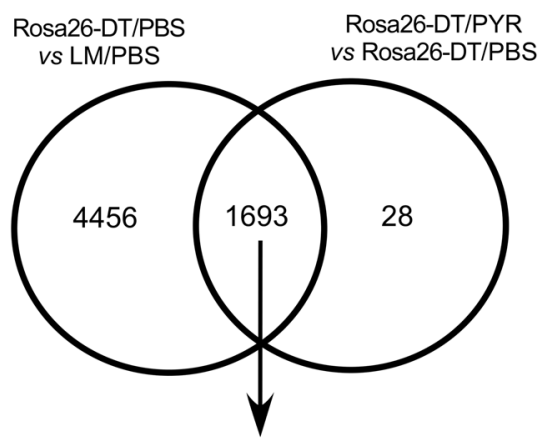

REVERSED BY PYR

1113 transcripts up and down 580 transcripts down and up
B

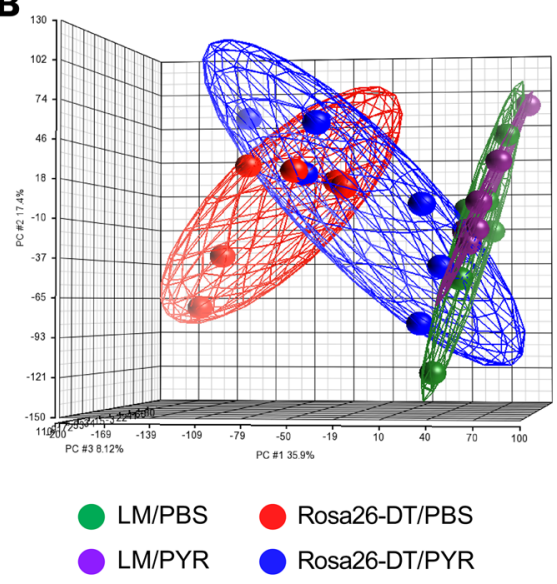

Figure 2. Transcriptional profiling of hearts after diphtheria toxin-mediated injury. (A) Venn diagram showing the number of transcripts that were differentially regulated in Rosa26-DT Micz-cre $^{-}$ (Rosa26-DT) versus LM hearts treated with diluent (PBS) and the transcripts that were differentially regulated in Rosa26-DTMIc2v-Cre hearts that were treated with pyridostigmine (PYR) or diluent on day 5 after diphtheria toxin (DT) injection ( $n=6$ hearts/group). Treatment with PYR normalized the expression of 1693 transcripts, of which 1113 were initially upregulated and 580 were initially downregulated. (B) Principal component analysis of changes in gene expression in hearts from LM and Rosa26-DTMic2v-Cre mice treated with diluent or PYR. (C) KEGG pathway analysis of the genes $(n=1693)$ that were normalized in the PYR-treated Rosa26-DTMicz-Cre mice. Blue, genes downregulated by PYR; red, genes upregulated by PYR.

mice, in order to ascertain which transcriptional networks were modulated by inhibiting acetylcholinesterase. As shown in Figure 2C, the majority of the KEGG pathways normalized in the Rosa26-DT ${ }^{\mathrm{Ml}}$ c2v-Cre/PYR comprised gene families belonging to inflammatory signaling pathways. We next examined changes in specific genes that were identified in the top 4 pathways that were most significantly regulated by PYR treatment. As shown by the heatmap in Supplemental Figure 2, all of the genes that were differentially regulated in the Rosa26-DT ${ }^{\text {Mlc2v-Cre }} / \mathrm{PBS}$

hearts were members of cytokine, cytokine receptor, chemokine, or chemokine receptor families. Moreover, save for 2 genes (Il15 and CtfI), the expression levels of all of the dysregulated genes shown in the heatmap were increased in the $\mathrm{DT}^{\mathrm{Mlc} 2 \mathrm{C}-\mathrm{Cre}} / \mathrm{PBS}$ hearts and partially normalized in the Rosa26-DT ${ }^{\mathrm{Mlc} 22-C r e} / \mathrm{PYR}$ mouse hearts. Although we did not explore which cell types contributed to the dysregulation of inflammatory genes in the Rosa26-DT ${ }^{\mathrm{Mlc} 2 \mathrm{~V}-\mathrm{Cre}} / \mathrm{PBS}$ hearts, it likely reflects changes in cardiomyocytes, endothelial cells, and infiltrating monocytes/macrophages. Viewed together, these data show that PYR treatment downregulates inflammatory signaling pathways following tissue injury.

Morphometric and histological analysis of PBS- and PYR-treated mouse hearts on day 5 revealed that both cardiac hypertrophy (Supplemental Figure 3, A and B) and cardiomyocyte cell area (Supplemental Figure 3, C-D) were significantly ( $P=0.03$ and $P<0.0001$, respectively) lower in Rosa26-DT ${ }^{\text {Mlc2v-Cre }} / \mathrm{PYR}$ hearts when compared with Rosa26-DT ${ }^{\mathrm{MI} c 2 v-C r e} / \mathrm{PBS}$ mouse hearts.

Treatment with pyridostigmine decreases influx of $\mathrm{CCR} 2^{+}$monocytes following cardiac injury. Given that PYR treatment reduced the infiltration of leukocytes in the heart following DT injury, we sought to determine which specific immune cell types were affected. Semiquantitative immunofluorescence staining of the heart on day 5 (Figure 3, A and B) showed that there was a significant $(P<0.0001)$ increase in the number of CD68 ${ }^{+}$ macrophages in the DT-treated Rosa26-DT ${ }^{\text {Mlc2v-Cre }} / \mathrm{PBS}$ hearts when compared with LM/PBS hearts. Howev- 
er, the salient finding shown by Figure $3, \mathrm{~A}$ and $\mathrm{B}$, is that the PYR treatment significantly $(P=0.002)$ reduced the number of $\mathrm{CD}^{+} 8^{+}$cells in the Rosa26-DT ${ }^{\mathrm{Mlc2v}-\mathrm{Cre}} / \mathrm{PYR}$ hearts when compared with Rosa26-DT ${ }^{\mathrm{Mlc} 2 \mathrm{C}-\mathrm{Cr}} /$ PBS hearts. To further explore these findings, we performed FACS analysis at day 5 after DT injection. The gating strategy for this FACS analysis is shown in Supplemental Figure 10. FACS analysis revealed that there was a significant increase in the number of $\mathrm{CD} 45^{+} \mathrm{Ly} 6 \mathrm{G}^{-} \mathrm{CD} 64^{+}$cells in the DT-injected Rosa26-DT ${ }^{\mathrm{Mlc} 2 \mathrm{~V}-\mathrm{Cre}}$ / PBS hearts in comparison with LM/PBS and LM/PYR hearts $(P=0.0004$ and $P=0.0002$, respectively, Figure $3 \mathrm{C}$ ). However, the salient finding shown by Figure $3 \mathrm{C}$ is that the number of $\mathrm{CD} 45^{+} \mathrm{Ly} 6 \mathrm{G}^{-} \mathrm{CD} 64^{+}$cells was significantly $(P=0.04)$ less in the Rosa26-DTMlc2v-Cre $/ P Y R$ hearts when compared with that in Rosa26-DTMlc2v-Cre/PBS hearts. In accordance with the findings obtained with immunofluorescence staining and FACS, the expression levels of $C d 68$ mRNA were significantly $(P=0.0003)$ increased in the Rosa26-DT ${ }^{\text {Mlc2v-Cre }} / \mathrm{PBS}$ mouse hearts when compared with LM/PBS hearts (Supplemental Figure 4A), whereas the expression levels of $C d 68$ mRNA were significantly $(P=0.04)$ decreased in the Rosa26-DT ${ }^{\mathrm{Mlc} 2 \mathrm{v}-\mathrm{Cre}} / \mathrm{PYR}$ hearts when compared with Rosa26-DT ${ }^{\text {Mlc2v-Cre }} / \mathrm{PBS}$ mouse hearts (Supplemental Figure 4A).

Both immunofluorescence staining (Supplemental Figure 5, A-B) and flow cytometry (Supplemental Figure 5C) showed that there was a significant $(P=0.0002$ and $P=0.01)$ increase in $\mathrm{Ly}_{6 \mathrm{G}}{ }^{+}$neutrophils in the DT-injured Rosa26-DT ${ }^{\text {Mc2v-Cre }} / \mathrm{PBS}$ hearts compared with LM/PBS hearts. Surprisingly, however, there was no significant difference in the number of $\mathrm{Ly}_{6} \mathrm{G}^{+}$cells detected by immunofluorescence $(P>0.99)$ or FACS $(P=0.82)$ in the DT-injured Rosa26-DT ${ }^{\mathrm{Mlc} 2 \mathrm{~V}-\mathrm{Cr}} / \mathrm{PYR}$ hearts compared with Rosa26-DT ${ }^{\mathrm{Mlc} 2 \mathrm{v}-\mathrm{Cr}} / \mathrm{PBS}$ hearts (Supplemental Figure 5, A-C). Similarly, we observed a significant $(P=0.03)$ increase in $\mathrm{CD}^{+} \mathrm{T}-\mathrm{lympho}$ cytes in the Rosa26-DT ${ }^{\text {Mlc2v-Cre }} / \mathrm{PBS}$ hearts when compared with LM/PBS hearts; however, treatment with PYR had no significant $(P>0.99)$ effect on the number $\mathrm{CD}^{+} \mathrm{T}$ cells in the Rosa26-DT ${ }^{\mathrm{Mlc} 2 \mathrm{~V}-\mathrm{Cr}} / \mathrm{PYR}$ hearts when compared Rosa26-DT ${ }^{\mathrm{Mlc} 2 \mathrm{-Cre}} / \mathrm{PBS}$ hearts (Supplemental Figure 5, D-E). Viewed together, these studies suggest that treatment with PYR selectively reduces the number of macrophages (CD68 ${ }^{+}$and $\mathrm{CD} 64^{+}$cells) in DT-injured hearts, without affecting the number of $\mathrm{Ly}_{6 \mathrm{G}}{ }^{+}$neutrophils and $\mathrm{CD}^{+} \mathrm{T}$ cells.

Recently, we have shown that the naive adult heart is populated by MHC-II ${ }^{\mathrm{hi} C C R} 2^{-}$and MHC-II ${ }^{\mathrm{lo}} \mathrm{CCR} 2^{-}$embryonic-derived tissue macrophages, whereas $\mathrm{MHC}-\mathrm{II}^{\mathrm{lo}} \mathrm{CCR} 2^{+}$monocytes and MHC-II ${ }^{\text {hi CCR }} 2^{+}$monocyte-derived macrophages populate the heart rapidly following tissue injury (15). To determine whether PYR treatment affected the stoichiometry of macrophage subsets in heart following tissue injury, we evaluated macrophage populations on day 5 after DT injection. As shown in Figure 3, D and E, there was a striking increase in the number of $\mathrm{MHC}-\mathrm{II}^{\mathrm{hi} C C R} 2^{+}(P<0.0001)$ and $\mathrm{MHC}-\mathrm{II}^{\mathrm{lo}} \mathrm{CCR} 2^{+}(P=0.0001)$ cells in the hearts of Rosa26-DT ${ }^{\mathrm{Mlc2v}-C r e} / \mathrm{PBS}$ mice, when compared with LM/PBS hearts, consistent with our previous findings (15). Remarkably, treatment with PYR significantly attenuated the increase in the number of $\mathrm{MHC}-\mathrm{II}^{\mathrm{lo}} \mathrm{CCR} 2^{+}$monocytes in Rosa26-DT ${ }^{\mathrm{Ml}}$ c2v-Cre/PYR hearts when compared with the Rosa26-DT ${ }^{\mathrm{Mlc2v}-\mathrm{Cr}} / \mathrm{PBS}$ hearts $(P=0.01)$. Although there was a numerical decrease in the MHC-II ${ }^{\mathrm{hi} C C R} 2^{+}$macrophages in the Rosa26-DT ${ }^{\mathrm{Mlc} 2 \mathrm{v}-\mathrm{Cre}} / \mathrm{PYR}$ hearts when compared with Rosa26-DT ${ }^{\mathrm{Mlc} 2 \mathrm{v}-\mathrm{Cre}} / \mathrm{PBS}$ hearts, these changes were not significantly different $(P=$ 0.22). Of note, $C c r 2$ mRNA expression levels were significantly increased $(P<0.0001)$ in Rosa26-DTMlc2v-Cre/PBS hearts in comparison with LM/PBS hearts, whereas the expression levels of $C$ cr $2 \mathrm{mRNA}$ were significantly reduced $(P=0.04)$ in the hearts treated with PYR (Rosa26-DT $\left.{ }^{\mathrm{Mlc} 2 \mathrm{v}-\mathrm{Cre}} / \mathrm{PYR}\right)$ in comparison with Rosa26-DT ${ }^{\mathrm{Mlc} 2 \mathrm{v}-\mathrm{Cre}} / \mathrm{PBS}$ hearts (Supplemental Figure 4B).

Mechanism for the pyridostigmine-induced decrease in monocyte-derived macrophages in DT-injured hearts. In order to determine the mechanism by which cholinesterase inhibition with PYR reduced the number of $\mathrm{MHC}-\mathrm{II}^{10} \mathrm{C}$ $\mathrm{CR}^{+}$monocytes in the heart, we asked whether there were differences in monocyte mobilization from the spleen or bone marrow following cardiac injury. As shown in Figure $3 \mathrm{~F}$, the number of $\mathrm{CD} 45^{+} \mathrm{F} 4 / 80^{+} \mathrm{Ly} 6 \mathrm{C}^{\mathrm{h}}$ ${ }^{i}$ Ly6G-CD3-CD19- circulating monocytes was significantly increased in the DT-injected (day 3) Rosa26-DTMlc2v-Cre $/ P B S(P=0.04)$ and Rosa26-DT ${ }^{\text {Mlc2v-Cre } / P Y R ~}(P=0.02)$ mice when compared with LM/PBS controls; however, the important finding shown by Figure $3 \mathrm{~F}$ was that there was no significant $(P=0.99)$ difference in the number of circulating monocytes between the Rosa26-DT ${ }^{\mathrm{Mlc} 2 \mathrm{v}-\mathrm{Cr}} / \mathrm{PBS}$ and Rosa26-DT ${ }^{\mathrm{Mlc} 2 \mathrm{~V}-\mathrm{Cre}} / \mathrm{PYR}$ mice. Similarly, although there was a significant $(P=0.02)$ decrease in the number of $\mathrm{CD}^{2} 5^{+} \mathrm{CD} 64^{+} \mathrm{Ly} 6 \mathrm{G}^{-} \mathrm{CD} 3$ CD19- splenic macrophages in the Rosa26-DT ${ }^{\text {Mlc2v-Cre }}$ PBS mice when compared with LM/PBS mice (Figure $3 \mathrm{G})$, there was no significant difference $(P=0.99)$ in the number of $\mathrm{CD} 45^{+} \mathrm{CD} 64^{+} \mathrm{Ly} 6 \mathrm{G}^{-} \mathrm{CD}^{-} \mathrm{CD} 19^{-}$splenic macrophages of the Rosa26-DT ${ }^{\mathrm{Mlc} 2 \mathrm{v}-\mathrm{Cr}} / \mathrm{PBS}$ and Rosa26-DT $\mathrm{Mlc2v}_{\mathrm{Cre}} / \mathrm{PYR}$ mice (day 3). Viewed together, these data suggest that PYR treatment inhibits the influx of monocytes in DTR-injured hearts without altering the mobilization of monocytes from the spleen and bone marrow. 
A

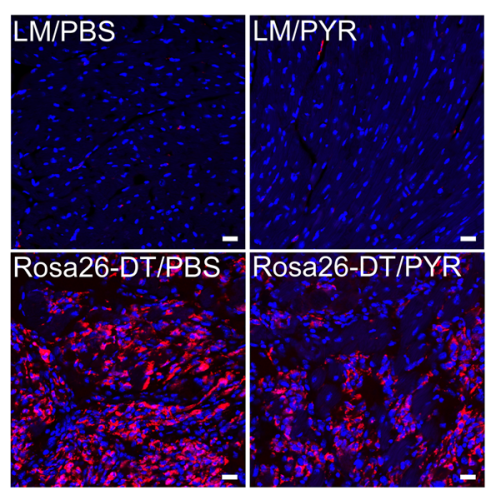

D

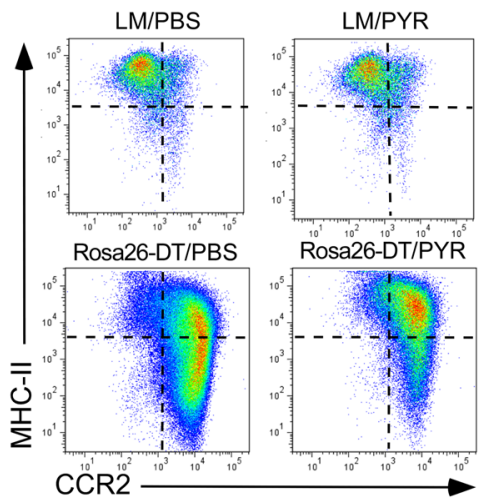

$\mathbf{F}$

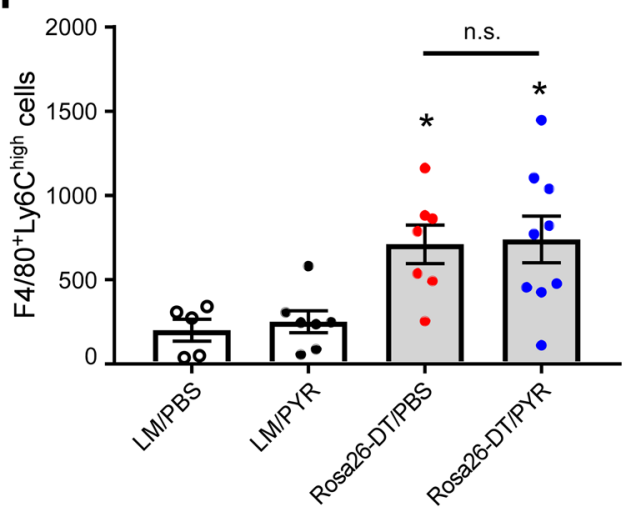

B

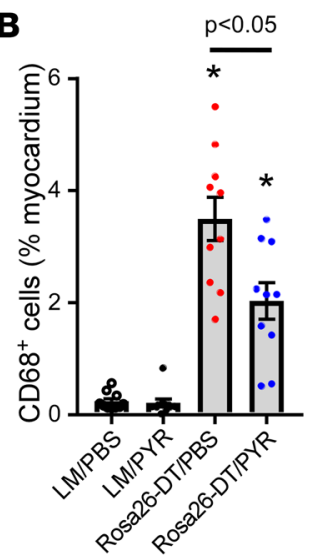

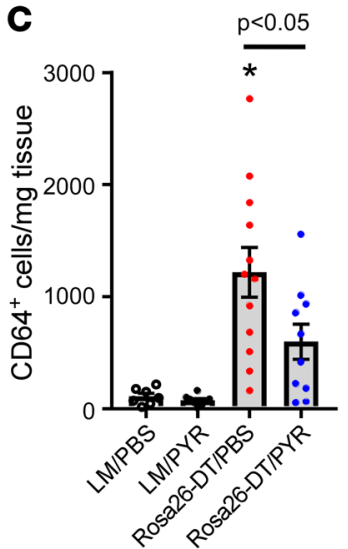

E

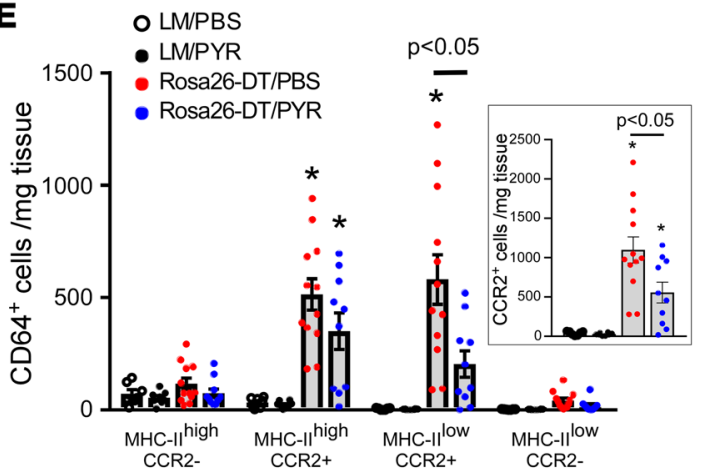

G

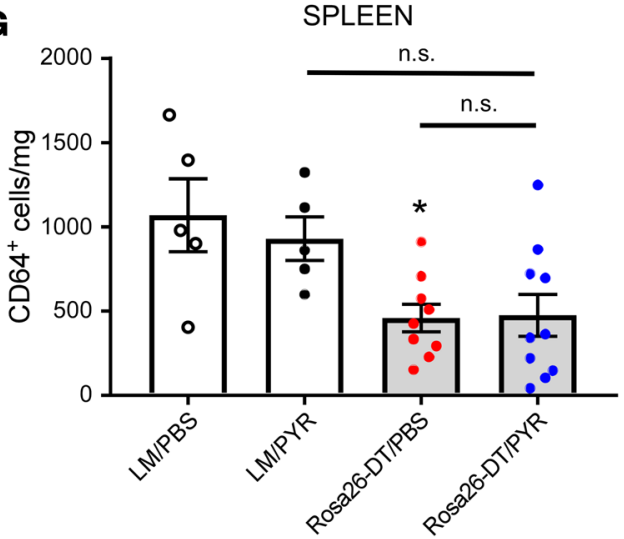

Figure 3. PYR treatment inhibits the influx of monocytes to the heart following diphtheria toxin-mediated injury. (A) Representative immunofluorescence images of CD68+ cells in the hearts of diphtheria toxin-injected (DT-injected) (day 5) Rosa26-DTMic2-cre (Rosa26-DT) and littermate control (LM) mice treated with PYR or diluent (PBS). Scale bar: $20 \mu \mathrm{m}$. (B) Group data for CD68+ staining in the hearts of DT-injected (day 5) Rosa26-DTMic2v-Cre and LM mice treated with PYR or diluent ( $n=10$ myocardium sections/group obtained from 5 hearts per condition). (C) Group data for CD64+ macrophages/monocytes in the hearts of DT-injected (day 5) Rosa26-DTMiczv-cre and LM mice treated with PYR or diluent by flow cytometry ( $n=7-12$ hearts/condition). (D) Representative FACS analysis of macrophage subsets (MHC-II ${ }^{\text {hi/lo }}, C_{C R 2^{+/}}$) in the hearts of DT-injected (day 5) Rosa26-DTMlc2v-Cre and LM mice treated with PYR or diluent. (E) Group data of macrophage subsets (MHC-I ${ }^{\text {hi/lo, }}$, CCR2 $^{+/}$) in the hearts of DT-injected (day 5) Rosa26-DTMlc2v-Cre and LM mice treated with PYR or diluent ( $n=7-12$ hearts/condition). (F) Group data for circulating F4/80+Ly6Chi monocytes in DT-injected (day 3) Rosa26-DTMlc2-cre and LM mice treated with PYR or diluent ( $n=5-9$ mice/ group). (C) Group data for CD64+ cells in the spleens of DT-injected (day 3) Rosa26-DTMic2v-re and LM mice treated with PYR or diluent ( $n=5-10$ mice/group). ${ }^{*} P$ $<0.05$ in comparison with LM/PBS and LM/PYR. $P$ values were calculated with 1-way ANOVA followed by the Tukey post hoc test.

Given that the transcriptional profiling data suggested that PYR treatment significantly reduced cytokine and chemokine expression in the heart (Supplemental Figure 2), we asked whether PYR treatment inhibited the early (i.e., day 3) expression of proinflammatory cytokines that regulate the expression of chemokine and chemokine receptors. Supplemental Figure 6, A-C, shows that the expression levels of Tnf, Il6, and Il1B 
mRNA were significantly increased in the hearts of Rosa26-DT ${ }^{\text {Mic2v-Cre }} / \mathrm{PBS}$ mice in comparison with LM/ PBS hearts $(P=0.02, P<0.0001$, and $P=0.01$, respectively) and that treatment with PYR significantly attenuated the expression levels of Tnf, Il6, and Ill $\beta$ mRNA when compared with Rosa26-DT ${ }^{\text {Mlc2v-Cre } / P B S}$ hearts $(P=0.03, P<0.0001$, and $P=0.02$, respectively). Next, we asked whether PYR treatment altered the expression levels of 2 chemokines released by CCR2 ${ }^{+}$cells that have been implicated in monocyte recruitment, namely CCL2 (Mcp-1) and CCL7 (Mcp-3; ref. 15). Figure 4, A and B, shows that the expression levels of $C c l 2(P<0.0001)$ and $C c l 7(P=0.007)$ mRNA increased significantly on day 3 in Rosa26-DT ${ }^{\mathrm{Mlc} 2 \mathrm{-Cre}} / \mathrm{PBS}$ hearts in comparison with LM/PBS hearts, whereas $C c l 2(P=0.0004)$ and $C c l 7(P=0.004)$ mRNA expression levels were significantly reduced in the hearts of the Rosa26-DT ${ }^{\text {Mlc2v-Cre }} / \mathrm{PYR}$ mice when compared with the hearts of Rosa26-DT ${ }^{\mathrm{MI} 2 \mathrm{v}-\mathrm{Cre}} / \mathrm{PBS}$ mice. Moreover, the expression levels of $C c l 2(P=0.86)$ and $C c l 7(P=0.98)$ mRNA in the Rosa26-DT ${ }^{\mathrm{Mlc} 2 \mathrm{v}-\mathrm{Cre}} / \mathrm{PYR}$ hearts were not significantly different from baseline values observed in LM/PYR hearts. Figure 4, C and D, shows that CCL2 $(P=0.0001)$ and CCL7 $(P=0.01)$ protein levels were both significantly increased in Rosa26-DT ${ }^{\text {Mlc2v-Cre }} / \mathrm{PBS}$ hearts when compared with LM/PBS hearts and that protein levels of CCL2 $(P=0.01)$ and CCL7 $(P=0.04)$ were decreased in Rosa26-DT ${ }^{\text {Mlc2v-Cre }} /$ PYR hearts compared with Rosa26-DT ${ }^{\mathrm{Mlc2v}-\mathrm{Cre}} / \mathrm{PBS}$ hearts (Figure $4, \mathrm{C}$ and D). There was a trend $(P=0.06)$ toward significance in the CCL2 protein levels in Rosa26-DT ${ }^{\text {Mc2v-Cre }} / \mathrm{PYR}$ hearts when compared with LM/PYR hearts. On the other hand, there was no significant difference in CCL7 $(P=0.54)$ protein levels in native LM/PYR and Rosa26-DT ${ }^{\text {Mlc2v-Cre } / P Y R ~ h e a r t s . ~}$

Given that CCR2 ${ }^{+}$macrophages are an important source of chemokines $(15,18)$, we investigated whether the decrease in myocardial levels of CCL2/7 in PYR-treated mice was secondary to decreased chemokine production by $\mathrm{CCR} 2^{+}$macrophages. Accordingly, we flow-sorted $\mathrm{MHC}-\mathrm{II}^{\mathrm{hi}} \mathrm{CCR} 2^{+}$macrophages and $\mathrm{MHC}_{\mathrm{II}}{ }^{\mathrm{lo}} \mathrm{CCR} 2^{+}$monocytes from Rosa26-DT ${ }^{\mathrm{Mlc} 2 \mathrm{v}-\mathrm{Cre}} / \mathrm{PBS}$ and Rosa26-DT ${ }^{\mathrm{Mlc2v}-\mathrm{Cre}} / \mathrm{PYR}$ hearts (day 3 ) in order to assess mRNA expression levels of $C c l 2 / 7$. As shown in Figure 4, E and F, there was a significant decrease in the mRNA expression levels of $C c l 2(P=0.03)$ and $C c l 7(P=0.02)$ in the $\mathrm{MHC}-\mathrm{II}^{\mathrm{hi}} \mathrm{CCR} 2^{+}$macrophages from the Rosa26-DT ${ }^{\text {Mlc2v-Cre }} / \mathrm{PYR}$ hearts when compared with Rosa26-DT ${ }^{\mathrm{Mlc2v}-\mathrm{Cr}} / \mathrm{PBS}$ hearts. Importantly, the mRNA expression levels of those chemokines were not significantly different in $\mathrm{MHC}-\mathrm{II}^{\mathrm{lo}} \mathrm{CCR} 2^{+}$ monocytes isolated from Rosa26-DT ${ }^{\mathrm{Mlc2} v-\mathrm{Cr}} / \mathrm{PBS}$ and Rosa26-DT ${ }^{\mathrm{Ml} 2 \mathrm{v}-\mathrm{Cre}} / \mathrm{PYR}$ hearts $(P=0.38$ and $P=0.12$, respectively, Figure 4, G and $\mathrm{H})$. These data demonstrate that PYR treatment selectively decreases $C c l 2$ and Ccl7 mRNA levels in MHC-II ${ }^{\text {hi CCR2 }}{ }^{+}$macrophages from DTR-injured hearts. Importantly, the difference in the expression of chemokines was not secondary to a difference in the number of macrophages and/or monocytes in the heart on day 3 after DTR injury (see Supplemental Figure 7 for details).

ACh inhibits CCL2 and TNF secretion by intraperitoneal macrophages. To determine the mechanism for the PYR-induced decrease in CCL2/7 expression, we examined the release of CCL2 in cultures of LPS-stimulated intraperitoneal macrophages (ipMACs), in the absence or presence of increasing concentrations of ACh $\left(10^{-6}\right.$ to $\left.10^{2} \mu \mathrm{M}\right)$. Treatment with ACh resulted in a significant ( $P=0.03$ by 1 -way ANOVA, Figure $5 \mathrm{~A})$ concentration-dependent decrease in CCL2 release in LPS-stimulated cultures. To determine whether the effects of ACh were through simulation of either nicotinic and/or muscarinic receptors, cultures of LPS-stimulated ipMACs were pretreated with increasing concentrations of nicotine $\left(10^{-6}\right.$ to $\left.10^{2} \mu \mathrm{M}\right)$ and muscarine $\left(10^{-6}\right.$ to $\left.10^{2} \mu \mathrm{M}\right)$. As shown in Figure $5 \mathrm{~A}$, pretreatment with nicotine and muscarine significantly $(P<0.0001$ and $P<0.0001$ by 1 -way ANOVA, respectively) inhibited CCL2 release in LPS-stimulated ipMACs in a concentration-dependent manner, suggesting that ACh inhibits CCL2 release through stimulation of both muscarinic and nicotinic receptors. On a molar basis, nicotine and muscarine were both significantly more potent with respect to inhibiting CCL2 release than ACh $(P<0.0001$ for nicotine and muscarine by 2 -way ANOVA). However, there was no significant difference ( $P=0.06$ by 2 -way ANOVA, Figure 5A) in CCL2 release between the nicotine or muscarine stimulated ipMACs cultures.

Given that ACh inhibits TNF release in circulating monocytes, and that TNF provokes increased expression of CCL2 (4), we asked whether ACh, nicotine, and muscarine inhibited TNF release from LPS-stimulated ipMACs. The important finding shown by Figure $5 \mathrm{~B}$ is that treatment with $\mathrm{ACh}$, nicotine, and muscarine significantly inhibited TNF release in a concentration-dependent manner $(P<0.0001$ for each comparison). Surprisingly, on a molar basis, inhibition of TNF secretion was not significantly different between $\mathrm{ACh}$ and nicotine $(P=0.86)$, whereas muscarine was significantly more potent on a molar basis than either $\mathrm{ACh}(P=0.0002$ by 2 -way ANOVA) or nicotine ( $P=0.0004$ by 2 -way ANOVA).

To determine which muscarinic receptors were involved in LPS-induced release of CCL2, we measured CCL2 release in LPS-stimulated ipMACs that were treated with muscarine, in the presence and absence of 
A

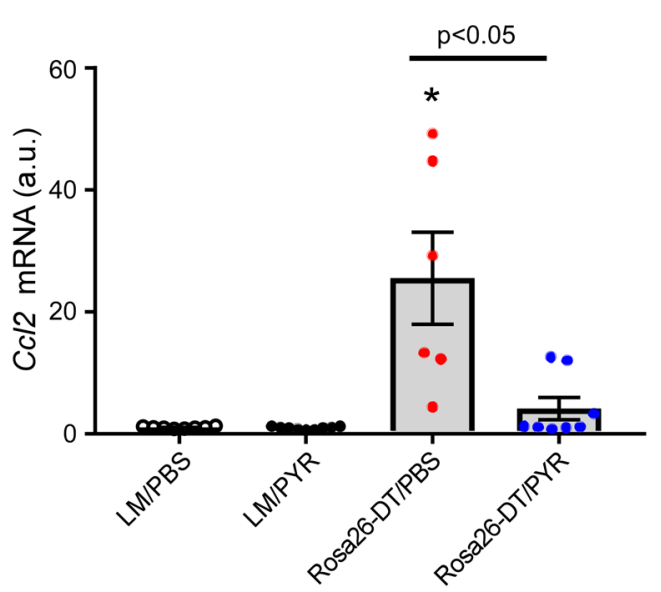

C

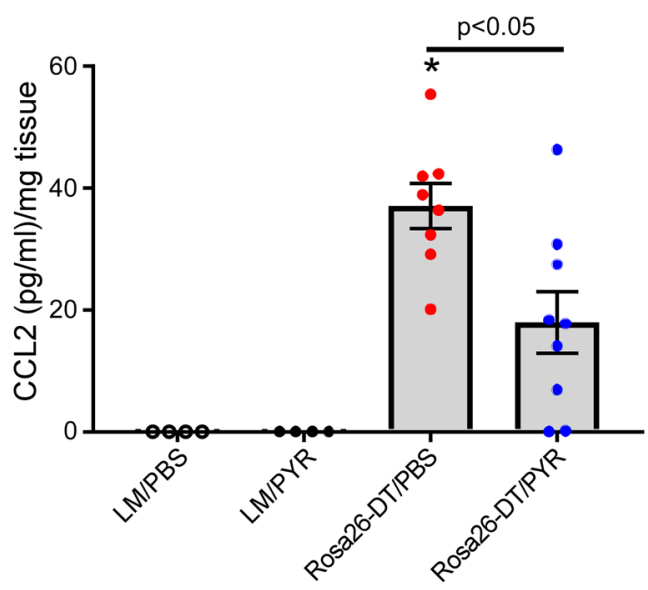

E MHC-II high
$\mathrm{CCR}^{+}$cells

$\mathbf{F}$

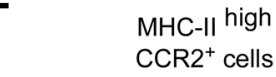

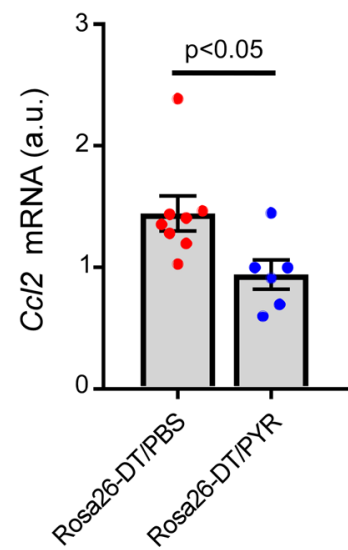

B

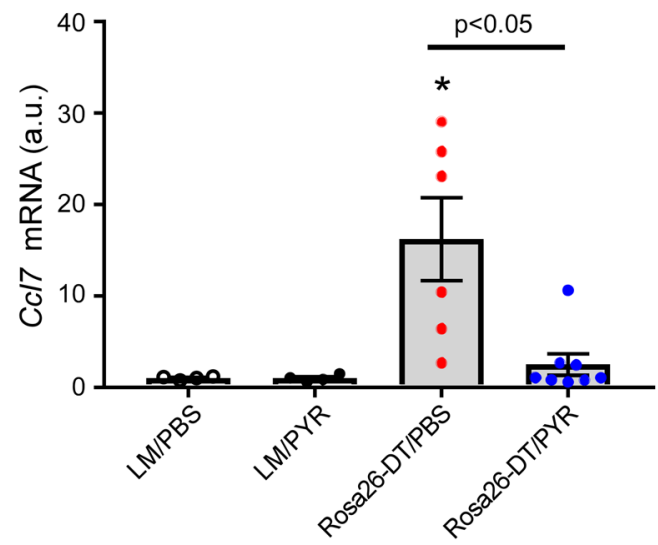

D

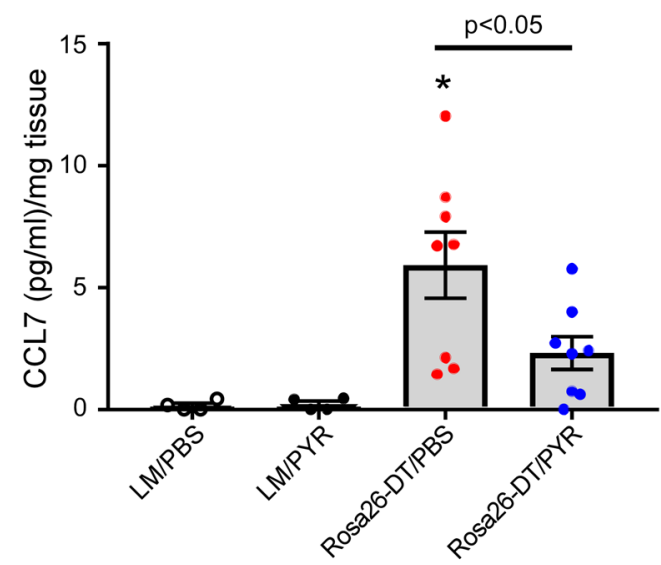

G
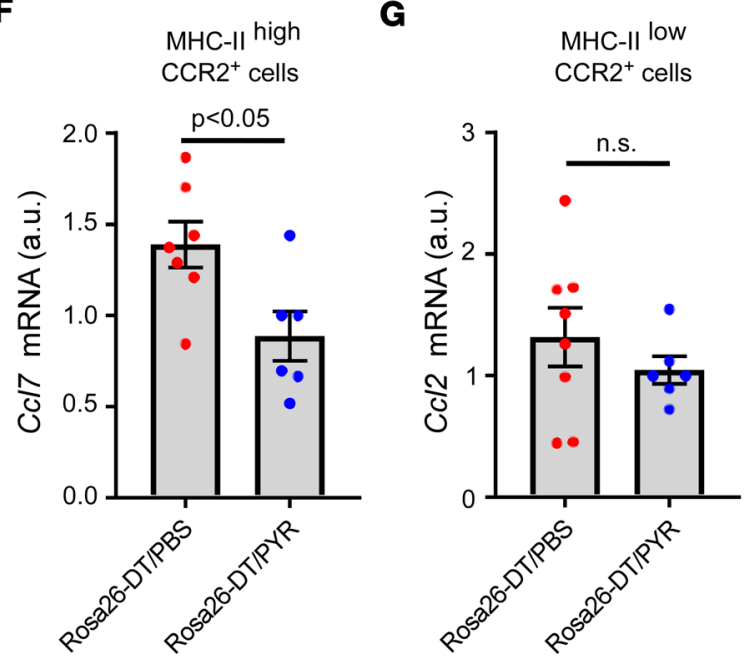

H MHC-II low
CCR2 $^{+}$cells

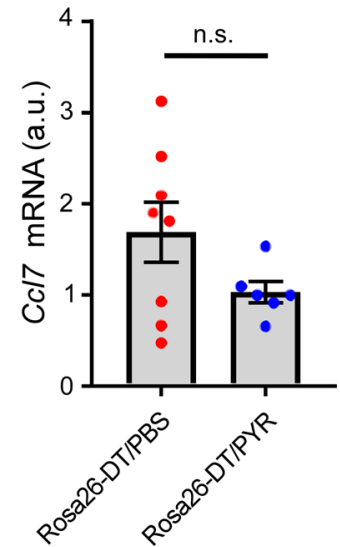

Figure 4. PYR treatment inhibits the expression of CCL2 and CCL7 chemokines in the heart following diphtheria toxin-mediated injury. (A and B) CCI2 and CCl7 mRNA expression in the hearts of diphtheria toxin-injected (DT-injected) (day 3) Rosa26-DTMlc2v-Cre (Rosa26-DT) and littermate control (LM) mice treated with PYR or diluent (PBS, $n=4-9$ hearts/group). (C and D) CCL2 and CCL7 protein levels in the hearts of DT-injected (day 3) Rosa26-DTMic2v-Cre and LM mice treated with PYR or diluent ( $n=4-9$ hearts/group). (E and F) Ccl2 and Ccl7 mRNA levels in flow-sorted MHC-IIh CCR2 ${ }^{+}$cells isolated from DT-injected (day 3) Rosa26-DTMic2-Cre mice treated with PYR or diluent ( $n=6-8$ hearts/group). (G and H) $C c / 2$ and Ccl7 mRNA levels in flow-sorted MHC-II'0CCR2 ${ }^{+}$cells isolated from DT-injected (day 3) Rosa26-DTMic2v-Cre mice treated with PYR or diluent ( $n=6-8$ hearts/group). ${ }^{*} P<0.05$ in comparison with LM/PBS and LM/ PYR. $P$ values were calculated with 1-way ANOVA followed by the Tukey post hoc test for A-D and Student's $t$ test for E-H. 
specific muscarinic receptor antagonists. In preliminary RNAseq studies (GEO accession GSE107776), we noted that M1 and M3 receptors were detectable in cultures of unstimulated ipMACs, whereas M2, M4, and M5 receptors were not detectable. Immunofluorescence staining confirmed the expression of M1 and M3 receptors in cells of cultured ipMACs (Figure 5C). Figure 5D shows that treatment with 4-DAMP, a M3 receptor antagonist, significantly attenuated $(P=0.01)$ the inhibitory effects of muscarine on CCL2 release, whereas treatment with telenzepine, a M1 receptor antagonist, did not significantly attenuate $(P=$ 0.7) the effects of muscarine on CCL2 secretion.

VAChT overexpression mimics the effects of the pyridostigmine in DT-injured hearts. To confirm the results with pharmacological inhibition of cholinesterase, we employed a genetic approach, wherein Rosa26-DT ${ }^{\mathrm{M}}$. Ic2v-Cre mice were backcrossed with hypercholinergic Chat-ChR2 mice (Rosa26-DT ${ }^{\text {Mlc2v-Cre } / C h a t-C h R 2) . ~ F i g u r e ~}$ 6A shows that Vacht mRNA expression was significantly $(P<0.0001)$ increased in the hearts of naive ChatChR2 mice when compared with LMs (day 3), consistent with a prior report (12). Although Vacht mRNA levels were significantly $(P<0.0001)$ increased in the hearts of the DT-injected Rosa26-DT ${ }^{\text {Mlc2v-Cre }}$ mice when compared with LMs, the levels of mRNA were not different $(P=0.99)$ from Chat-ChR2 mouse hearts. However, the important finding shown in Figure 6A is that there was a significant approximately 3-fold increase $(P<0.0001)$ in Vacht mRNA levels in the hearts of the DT-injected Rosa26-DT ${ }^{\text {Mlc2v-Cre } / C h a t-C h R 2 ~}$ mice when compared with DTR-injected Rosa26-DT ${ }^{\mathrm{Mlc2v}-\mathrm{Cre}}$ mice, consistent with a prior study that demonstrated that the cardiac Vacht mRNA expression is dynamic and increases with stress (19). We also performed immunohistochemical staining of VAChT protein in the hearts of DT-injected Rosa26-DT ${ }^{\mathrm{Mlc} 2 \mathrm{v}-\mathrm{Cr}}$ and Rosa26-DT ${ }^{\mathrm{Mlc} 2 \mathrm{v}-\mathrm{Cre}} /$ Chat-ChR2 mice. As shown by the representative immunofluorescence images in Figure 6B and the group data in Figure 6C, there was a significant $(P=0.02)$ increase in VAChT protein in the hearts of the Rosa26-DTMlc2v-Cre $/$ Chat-ChR2 mice when compared with the Rosa26-DT ${ }^{\text {Mlc2v-Cre }}$ mice.

To determine whether increased VAChT expression reduced cardiac inflammation, we measured both Ccl2/7 mRNA expression on day 3 as well as the number of CD68 ${ }^{+}$cells in LM, Chat-ChR2, Rosa26-DT ${ }^{\mathrm{Ml}-}$ c2v-Cre , and Rosa26-DT Mlc2v-Cre / Chat-ChR2 mouse hearts on days 3 and 5. As shown in Figure 6, D and E, the expression of $C c l 2 / 7 \mathrm{mRNA}$ was significantly increased $(P=0.0001$ for both $C c l 2$ and $C c l 7)$ in DT-injected Rosa26-DT ${ }^{\text {Mlc2v-Cre }}$ mouse hearts in comparison with naive LM mice (day 3). Importantly, $C c l 2$ and $C c l 7$

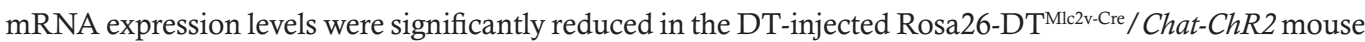
hearts when compared with the Rosa26-DT ${ }^{\mathrm{Ml} 2 \mathrm{v}-\mathrm{Cre}}$ mouse hearts $(P=0.04$ and $P=0.01$, respectively). However, the expression levels of $C c l 2$ and $C c l 7$ mRNA in Rosa26-DT ${ }^{\text {Mlc2v-Cre } / C h a t-C h R 2 ~ h e a r t s ~ r e m a i n e d ~}$ significantly higher than in naive LM or Chat-ChR2 hearts $(P=0.01$ and $P=0.03$, respectively, Figure 6, D and E). Analogous to the observations in the DT-injected PYR-treated Rosa26-DT ${ }^{\text {Mlc2v-Cre }}$ mice, Tnf mRNA expression was significantly $(P=0.003)$ reduced in the DT-treated Rosa26-DT ${ }^{\text {MIc2v-Cre } / C h a t-C h R 2 ~}$ hearts when compared with Rosa26-DT ${ }^{\mathrm{Mlc} 2 \mathrm{v}-\mathrm{Cre}}$ mouse hearts (Supplemental Figure 8A). Moreover, there was a significant decrease ( $P=0.0001$, Figure $6, \mathrm{~F}$ and $\mathrm{G})$ in the number of CD68 ${ }^{+}$cells in the DT-injected

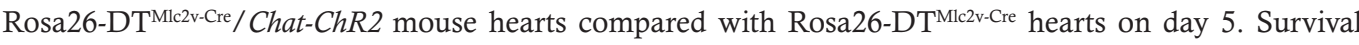
studies were not performed on the Chat-ChR2 and Rosa26-DT ${ }^{\mathrm{Mlc} 2 \mathrm{v}-\mathrm{Cre}} /$ Chat-ChR2 mice.

Importantly, the decrease in the number of $\mathrm{CD}^{2} 8^{+}$cells in the Rosa26-DT ${ }^{\mathrm{Mlc} 2 \mathrm{~V}-\mathrm{Cr}} /$ Chat-ChR2 hearts on day 5 was not secondary to differences in the initial degree of cell injury between Rosa26-DT ${ }^{\text {Mlc2v-Cre }}$ and Rosa26-DT ${ }^{\text {Mlc2v-Cre } / C h a t-C h R 2 ~ m i c e ~ o n ~ d a y ~} 3$ (see Supplemental Figure 8B). Quantification of CD68 ${ }^{+}$cells in the heart by immunofluorescence on day 3 after DT injection showed that there was a significant increase in the number of $\mathrm{CD}^{+} 8^{+}$cells in both Rosa26-DT ${ }^{\mathrm{Mlc2v}-\mathrm{Cre}}(P=0.0003)$ and Rosa26-DT ${ }^{\mathrm{Mlc} 2 \mathrm{v}-\mathrm{Cre}} /$ Chat-ChR2 $(P$ $=0.0005)$ hearts when compared with baseline values; however, there was no significant difference $(P=$ $0.9)$ between the Rosa26-DT ${ }^{\text {Mlc2v-Cre }}(P=0.0003)$ and Rosa26-DT ${ }^{\text {Mlc2v-Cre }}$ Chat-ChR hearts (Supplemental Figure 8, C and D). Viewed together, these data are consistent with the conclusion that increased VAChT expression leads to decreased numbers of $\mathrm{CD} 68^{+}$macrophages in the heart following tissue injury (day 5), at least in part, through inhibition of $C c l 2 / 7$ chemokine release (day 3 ).

\section{Discussion}

The results of this study, in which we used pharmacologic and genetic strategies to increase nonneuronal cholinergic signaling in the heart in the setting of tissue injury, show that nonneuronal cholinergic signaling confers beneficial immunomodulatory effects in the heart by decreasing the influx of proinflammatory $\mathrm{CCR}^{+}$monocytes. Four interrelated lines of evidence support this statement. First, treatment of DT-injected Rosa26-DT ${ }^{\text {Mlc2v-Cre }}$ mice with PYR, a cholinesterase inhibitor that does not cross the blood-brain barrier, 
A

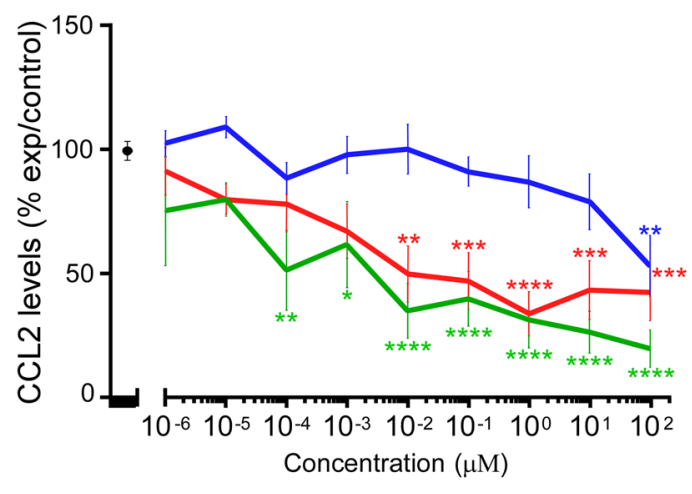

- LPS — ACh — Nicotine - Muscarine
B

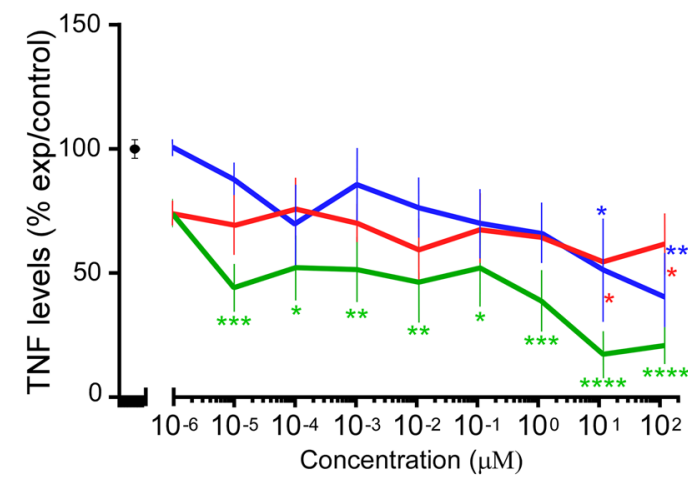

D

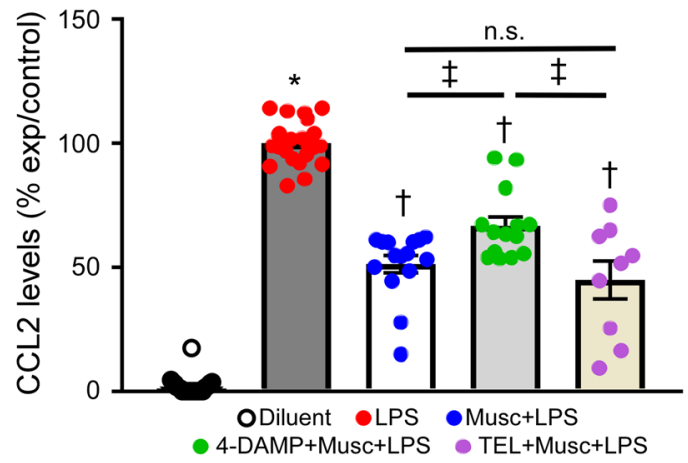

Figure 5. CCL2 and TNFs secretion in cultures of lipopolysaccharide-stimulated intraperitoneal macrophages. (A) CCL2 protein levels in the supernatant of cultures of lipopolysaccharide-stimulated (LPS-stimulated) intraperitoneal macrophages (ipMACs) treated in the presence and absence of increasing concentrations of acetylcholine (ACh), muscarine, and nicotine ( $n=3-14$ biological replicates per group). (B) TNF protein levels in the supernatant of cultures of LPS-stimulated ipMACs treated in the presence and absence of increasing concentrations of acetylcholine, muscarine and nicotine $(n=3-9$ biological replicates per group). Data are shown as percentage of experimental control (LPS) per concentration \pm SEM, analyzed by 2-way ANOVA. For $\mathbf{A}$ and $\mathbf{B},{ }^{*} P<0.05,{ }^{* *} P<0.01,{ }^{* *} P<0.001,{ }^{* * *} P \leq 0.0001$ in comparison with LPS. The graphs were produced using GPLOT procedure of SAS. For each concentration in the $x$ axis, the graph reports on the $y$ axis the mean analyte level expressed as percentage of control (LPS) \pm SEM. (C) Representative immunofluorescence staining of M1 and M3 ACh receptors in cultures of ipMACs. Scale bar: $20 \mu \mathrm{m}$. Green, M1 or M3; blue, DAPI. (D) CCL2 protein levels in the supernatant of cultures of LPS-stimulated ipMACs in the presence and absence of muscarine, 4-DAMP (M3 receptor antagonist), and telenzepine (M1 receptor antagonist, $n=9-24$ biological replicates per group). Diluent, PBS. For $\mathbf{D},{ }^{*} P<0.0001$ in comparison with LPS-treated cells; ${ }^{\dagger} P<0.0001$ in comparison with diluent (nonstimulated ipMACs) and LPS-stimulated cells; ${ }^{\ddagger} P<0.01$ in comparison with cells treated with 4 -DAMP, muscarine, and LPS. $P$ value was calculated using 2-way ANOVA using generalized linear models procedure with the option ADJUST = TUKEY to account for any missing values in $\mathbf{A}$ and B and with 1-way ANOVA followed by the Tukey post hoc test for $\mathbf{D}$.

resulted in decreased mortality (Figure 1A) and decreased inflammation in the heart (Figure 1, E and F) when compared with diluent-treated controls. Importantly, the beneficial effects of PYR were not secondary to differences in initial tissue injury in the PYR- and diluent-treated Rosa26-DT ${ }^{\text {Mic2v-Cre }}$ mice (Figure 1, B-D). Although the mode of DT-induced death was not explored in the present study, nonneuronal cholinergic signaling has been shown to exert antiarrhythmic properties in the heart (20). Second, transcriptional profiling using RNAseq revealed that treatment with PYR partially attenuated the dysregulated inflammatory signaling pathways in DT-injured Rosa26-DT ${ }^{\mathrm{Mlc2v}-\mathrm{Cre}}$ hearts (Figure 2). As shown in Supplemental Figure 2, PYR treatment partially normalized genes belonging to the cytokine, cytokine receptor, chemokine, and chemokine receptor families when compared with diluent-treated controls. Third, PYR treatment significantly decreased the number of $\mathrm{CD}^{+} 8^{+}$cells in DT-injected Rosa26-DT ${ }^{\text {Mlc2v-Cre }}$ hearts (Figure 3), whereas it had no effect on the influx of $\mathrm{Ly}_{6} \mathrm{G}^{+}$neutrophils or $\mathrm{CD}^{+} \mathrm{T}$ cells in DT-injected Rosa26-DT ${ }^{\mathrm{MI} 22 \mathrm{~V}-\mathrm{Cre}}$ hearts (Supplemental Figure 5). Although PYR treatment had no effect on the number of MHC-II ${ }^{10} \mathrm{CCR} 2^{-}$, MHC-II${ }^{\text {hi CCR2 }}{ }^{-}, \mathrm{MHC}_{-} \mathrm{II}^{\mathrm{hi}} \mathrm{CCR} 2^{+}$, and $\mathrm{MHC}-\mathrm{II}^{\mathrm{lo}} \mathrm{CCR} 2^{+}$macrophages/monocytes in naive hearts, PYR treatment 
A
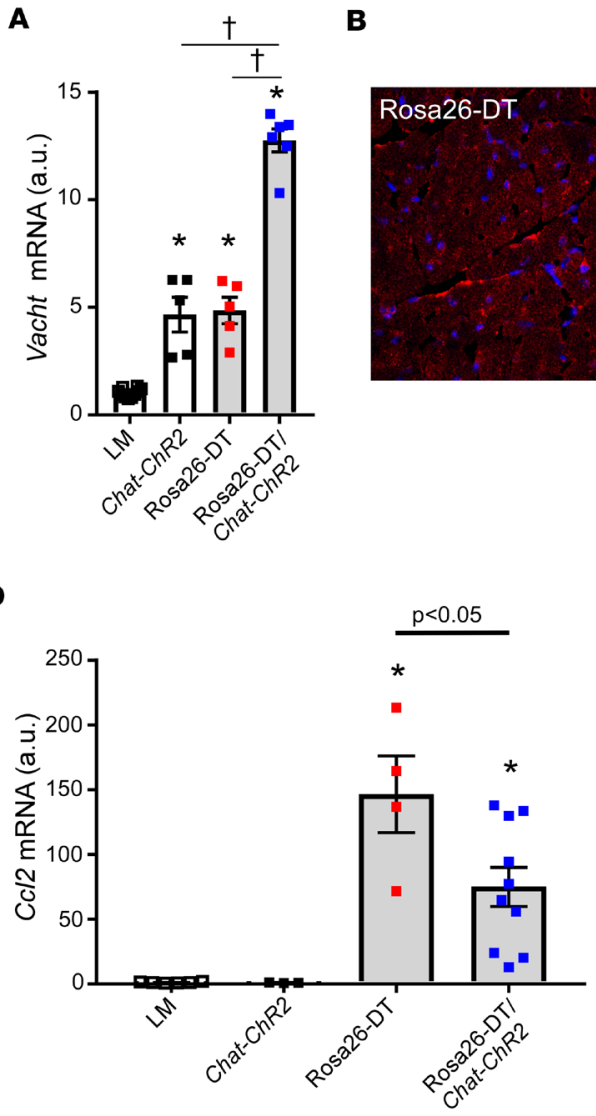

$\mathbf{F}$

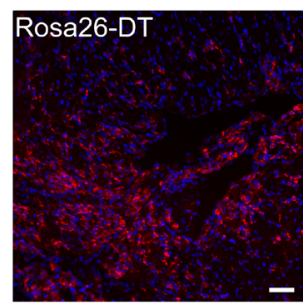

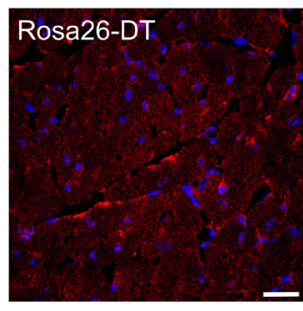
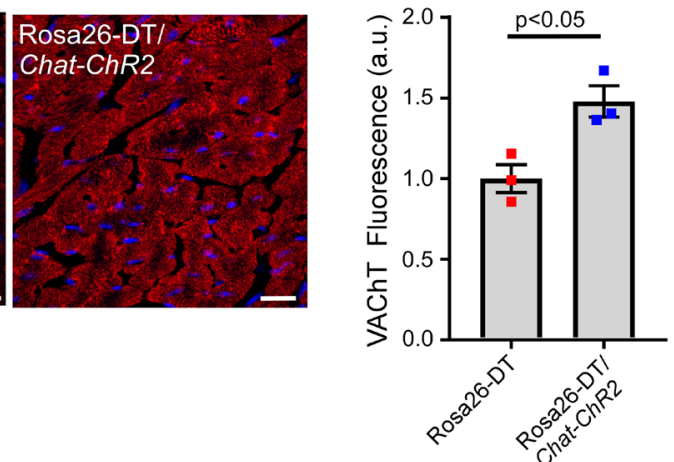

$\mathbf{E}$

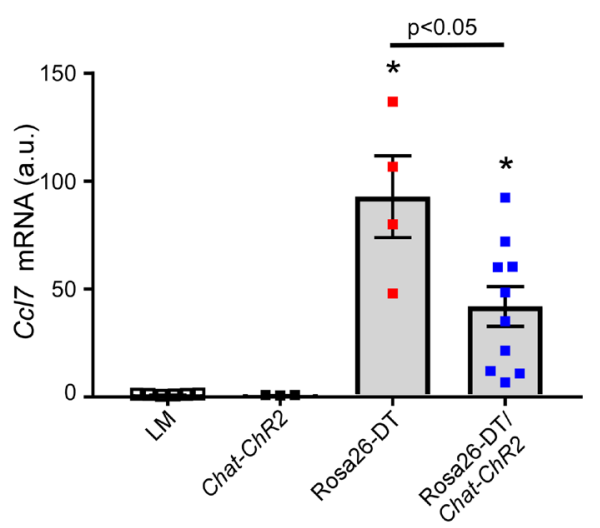

G

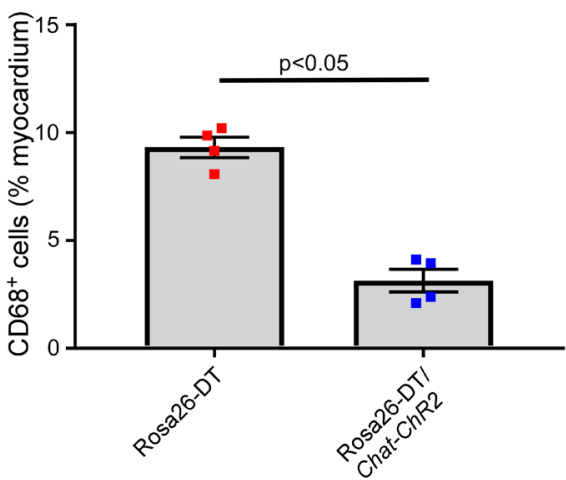

Figure 6. Effect of VAChT overexpression in the heart following diphtheria toxin-mediated injury. (A) Vacht mRNA levels in the hearts of littermate

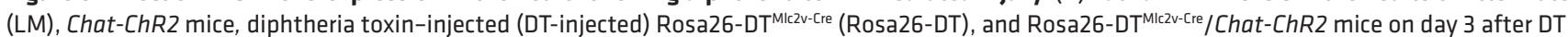
injection ( $n=5-10$ hearts/group). (B) Representative VAChT immunofluorescence staining in DT-injected (day 3) Rosa26-DT ${ }^{\text {Mic2v-Cre and Rosa26-DT }}{ }^{\text {Ml- }}$ c2v-cre/Chat-ChR2 hearts. Scale bar: $20 \mu \mathrm{m}$. (C) Group data of VAChT expression by immunofluorescence staining in DT-injected (day 3) Rosa26-DTMlc2v-Cre and Rosa26-DTMlc2v-Cre/Chat-ChR2 hearts ( $n=3$ hearts/condition). (D and E) Ccl2 and Ccl7 mRNA expression in the hearts of DT-injected (day 3) LM, Chat-ChR2, Rosa26-DTMic2v-Cre, and Rosa26-DTMlc2v-Cre/Chat-ChR2 mice ( $n=3-10$ hearts/group). (F) Representative immunofluorescence staining of CD68 ${ }^{+}$cells in DT-injected Rosa26-DT ${ }^{\text {Mic2v-Cre }}$ and Rosa26-DT ${ }^{\text {Mic2v-Cre } / C h a t-C h R 2 ~ h e a r t s ~ o n ~ d a y ~} 5$ after DT administration. Scale bar: $50 \mu \mathrm{m}$. (G) Group data of CD68 ${ }^{+}$cells in

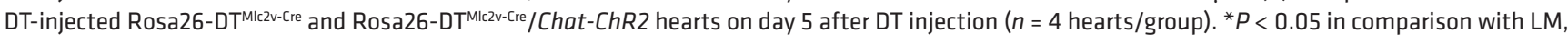
${ }^{\dagger} P<0.05$ in comparison with Rosa26-DTMlc2v-Cre $/$ Chat-ChR2. P values were calculated with 1-way ANOVA followed by the Tukey post hoc test for A, D, and $\mathbf{E}$ and Student's $t$ test for $\mathbf{C}$ and $\mathbf{G}$.

significantly reduced the influx of $\mathrm{MHC}-\mathrm{II}^{\mathrm{lo}} \mathrm{CCR} 2^{+}$monocytes (Figure 3) following DT-induced tissue injury. The decrease in infiltration of $\mathrm{MHC}-\mathrm{II}{ }^{\mathrm{Io}} \mathrm{CCR} 2^{+}$monocytes was not secondary to decreased mobilization of splenic macrophages, nor decreased circulating monocytes in the PYR-treated mice (Figure 3). Remarkably, PYR treatment significantly reduced the expression of proinflammatory cytokines ( Tnf, Ill $\beta$, and Il6) in DT-injected Rosa26-DT ${ }^{\mathrm{Ml} 2 \mathrm{v}-\mathrm{Cre}}$ hearts as well as the mRNA and protein levels of $2 \mathrm{CC}$ chemokines that have 
been implicated in monocyte recruitment (Figure 4), namely CCL2 (Mcp-1) and CCL7 (Mcp-3; ref. 15, 18). Flow sorting of CCR2 ${ }^{+}$cells from Rosa26-DT ${ }^{\text {Mlc2v-Cre }} / \mathrm{PBS}$ and Rosa26-DT ${ }^{\text {Mlc2v-Cre }} / \mathrm{PYR}$ hearts revealed that there was a significant decrease in the mRNA expression levels of $C c l 2$ and $C c l 7$ in $\mathrm{MHC}-\mathrm{II}^{\text {hi }} \mathrm{CCR} 2^{+}$tissue macrophages (Figure 4), suggesting that increased $\mathrm{ACh}$ availability decreased the recruitment of $\mathrm{MHC}-\mathrm{II}^{\mathrm{lo}} \mathrm{C}$ $\mathrm{CR}^{+}$monocytes, at least in part, through a mechanism that involves decreased expression of $\mathrm{Ccl} 2$ and $\mathrm{Ccl} 7$ by $\mathrm{MHC}-\mathrm{II}{ }^{\mathrm{hi}} \mathrm{CCR} 2^{+}$macrophages. However, we cannot exclude the formal possibility that the PYR-induced decrease in the expression of cardiomyocyte-derived $T n f, I l 1 \beta$, and $I l 6$ may have contributed to the decrease in influx of $\mathrm{MHC}-\mathrm{II}^{\mathrm{lo}} \mathrm{CCR} 2^{+}$macrophages/monocytes. In vitro studies in ipMACs demonstrated that stimulation of nicotinic receptors inhibited secretion of CCL2, consistent with prior reports that have suggested that ACh exerts antiinflammatory effects through activation of nicotinic receptors $(4,5)$. However, we also observed that stimulation of the M3 ACh receptors on ipMACs inhibited CCL2 secretion, thus revealing an unexpected role for muscarinic receptors in nonneuronal cholinergic signaling in macrophages (Figure 5). Fourth, backcrossing Rosa26-DT ${ }^{\text {Mlc2v-Cre }}$ mice with a transgenic mouse line that constitutively overexpresses VAChT (Chat-ChR2 mice), resulted in decreased expression of $\operatorname{Tnf}$ and Ccl2/7 mRNA (day 3) as well as a decrease in the number of $\mathrm{CD}^{+} 8^{+}$cells in DT-injured Rosa26-DT ${ }^{\mathrm{Mlc2v}-C r e} /$ Chat-ChR2 mouse hearts (day 5), consistent with our observations in PYR-treated Rosa26-DT ${ }^{\text {Mlc2v-Cre }}$ mouse hearts (Figure 6 and Supplemental Figure 8). Taken together, these studies suggest that the nonneuronal cholinergic system plays an important immunoregulatory role in the heart, at least in part, by inhibiting the expression of CC chemokines and recruitment of CCR2 $2^{+}$monocytes to the injured heart.

Cholinergic signaling in the heart. Whereas sympathetic regulation of the heart has been studied extensively, far less is known with respect to the role of cholinergic signaling in the heart, especially regarding the regulation of cardiac inflammation. There are two major sources of cholinergic signaling in heart: neuronal cholinergic signaling mediated by the release of ACh from the vagus nerve, and the more recently described nonneuronal cholinergic system, mediated by the release of ACh by cells that reside in the heart, including cardiomyocytes $(11,21)$.

The importance of neuronal cholinergic signaling in the heart has been suggested by gain-of-function studies, which demonstrated the beneficial effects of transection of the vagal nerve in animal models of cardiac injury (22) as well as direct stimulation of the vagus nerve in animal and human heart failure (23, 24). Loss-of-function studies in knockout mice that lack the M2 ACh receptor demonstrated that these mice are more susceptible to adrenergic stress (25). It has also been proposed that the neuronal cholinergic system plays an important immunomodulatory role sepsis and tissue injury. Indeed, recent studies suggest that peripheral afferent vagal nerves transmit danger signals from the periphery (sensory arm) to integrative regions within the brain stem that reciprocally activate vagal efferent fibers (motor arm) that terminate in the spleen and other immune tissues (the cholinergic antiinflammatory pathway; ref. 26). Activation of splenic efferent fibers stimulates splenic $\mathrm{CD} 4^{+} \mathrm{T}$ cells to release $\mathrm{ACh}$, which binds to $\alpha 7$ nicotinic receptors on splenic macrophages and inhibits TNF release (6). However, it bears emphasizing that studies that have employed vagal nerve stimulation or vagal nerve transection in studying the role of neuronal cholinergic signaling in the heart are all confounded by the reciprocal nature of parasympathetic and sympathetic signaling the heart (27). That is, transection of the vagal nerve results in a reflex increase in sympathetic nerve trafficking to the heart, which is sufficient activate a brisk immune response (28), whereas direct vagal nerve stimulation results in a reflex decreased in sympathetic nerve trafficking to the heart, which would decrease the extent of tissue injury and immune cell activation following myocardial injury (28). Accordingly, it becomes difficult to separate the direct effects of vagal nerve stimulation from the countervailing effects mediated by the sympathetic nervous system.

The evolutionarily conserved nonneuronal cholinergic system, which has been detected in bacteria, algae, protozoa, primitive plants, and invertebrate and vertebrate species predates the development of neuronal cholinergic signaling (21). Germane to this discussion, recent studies have revealed an important homeostatic role for nonneuronal cholinergic signaling in the heart $(9,11,12,29)$. Indeed, ACh that is released by cardiomyocytes attenuates the deleterious effects of excessive adrenergic signaling (11), reduces the levels of oxidative stress in cardiomyocytes (29), and modulates energy metabolism by enhancing glucose utilization in the heart (22). Remarkably, however, the immunomodulatory role of nonneuronal cholinergic signaling is not well understood. Indeed, a prior study in rats that underwent open chest occlusion of the LAD reported that pretreatment with PYR had no effect of "M1" macrophages or CD4" and $\mathrm{CD}^{+} \mathrm{T}$ cells on day 3 after infarction, whereas PYR treatment resulted in a significant increase in CD206 
"M2" macrophages and $\mathrm{CD} 4{ }^{+} \mathrm{CD} 25^{+} \mathrm{FOXP}^{+} \mathrm{T}$ lymphocytes in the infarct and periinfarct zones of the PYR-treated rats (30). The authors concluded that increased cholinergic signaling resulted in increased recruitment of antiinflammatory cells to the heart and that the increased influx of FOXP3 ${ }^{+}$Tregs resulted $^{2}$ in M1/M2 polarization toward increased M2 cells. These results differ from those presented herein, where treatment with PYR resulted in decreased recruitment of proinflammatory CCR2 ${ }^{+}$monocytes (formerly referred to as M1 cells) to the heart. Although the reasons for the discrepancy between the two studies is not known, it may relate to differences in species chosen, experimental injury models, or differences in methods for characterizing the immune cell types.

Conclusion. Although prior studies have suggested an important immunomodulatory role for neuronal cholinergic signaling mediated by the vagus nerve (6), the immunomodulatory of role of the nonneuronal signaling is far less well-defined. Here, we show that nonneuronal cholinergic signaling confers beneficial immunomodulatory effects in the heart by decreasing the influx of proinflammatory CCR $2^{+}$monocytes following tissue injury. However, these studies do not exclude a potential role for the neuronal cholinergic system in terms of modulating inflammatory responses following tissue injury. Our findings with respect to the immunomodulatory role of nonneuronal ACh signaling in the heart may have therapeutic import, given that treatment with anticholinesterase inhibitors decreased the risk of myocardial infarction and death in patients with Alzheimer's disease (31). Apart from the potential therapeutic implications of our findings, this study raises important questions with respect to the physiological role of neuronal and nonneuronal cholinergic systems in the heart. While the extant literature clearly suggests that the cholinergic antiinflammatory pathway acts reflexively to dampen the levels inflammatory mediators in the periphery, two distinct lines of evidence suggest that neuronal cholinergic system is less important in terms of modulating local immune responses in myocardial tissue. First, in contrast to the extensive sympathetic innervation of the heart, parasympathetic innervation of the heart is comparatively less and is greater in atrial than in ventricular tissue (32). Second, prior studies have shown that immune cells release preformed ACh through activation of Toll-like receptors, which we and others have shown are stimulated by DAMPs released by necrotic cardiomyocytes $(13,33)$. Thus, the parasympathetic nervous system is not necessarily required for mediating local antiinflammatory responses in the heart. Viewed together, these observations suggest the intriguing possibility that the nonneuronal cholinergic system, analogous to the innate immune system, may act as an "early warning" system that modulates local inflammatory responses to tissue injury in the heart, whereas the neuronal cholinergic system, which links the parasympathetic autonomic nervous system with peripheral lymphoid tissues, may serve to regulate systemic inflammatory responses by activating adaptive immune cells residing in lymphoid tissues. Given the recent interest in modulating inflammatory responses through implantable nerve stimulators ("bioelectric medicine"; ref. 34), as well as drugs that modulate $\alpha 7 \mathrm{nAChR}$ signaling, it will be important to clarify how the neuronal and nonneuronal cholinergic systems interact in order to develop effective treatment strategies designed to target the cardio-neuro-immune axis.

\section{Methods}

\section{Transgenic mouse lines}

The DT-based mouse model for selectively ablating cardiomyocytes has been described previously (15). Briefly, Mlc2v-Cre mice (C57BL/6J, The Jackson Laboratory) were crossed to Rosa26-DT mice (C57BL/6J)

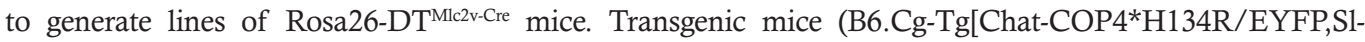
c18a3)6Gfng/J; ref. 35]) overexpressing the channelrhodopsin-2 (ChR2) gene under control of the ChAT gene (referred to herein as Chat-ChR2 mice) were provided by Marco Prado (Robarts Research Institute, University of Western Ontario, London, Ontario, Canada). Because the locus for the VAChT lies between the first and second introns of the ChAT gene, Chat-ChR2-transgenic mice overexpress both VAChT mRNA and protein (36). Moreover, prior studies have shown that VAChT protein levels are increased in cardiomyocytes and that ACh secretion is also increased in cardiac tissue isolated from the Chat-ChR2-transgenic mice (12).

To determine whether systemic overexpression of $V A C h T$ would attenuate the effects of DT-mediated cardiac injury in Rosa26-DT ${ }^{\mathrm{Mlc} 2 \mathrm{v}-\mathrm{Cre}}$ mice, we first backcrossed $\mathrm{Mlc} 2 \mathrm{v}-\mathrm{Cre}^{+/-}$and $\mathrm{Chat}$-ChR2 ${ }^{+/-}$mice. The resultant double-transgenic male mice ( $\left.M l c 2 v-\mathrm{Cre}^{+/-} / \mathrm{Chat}-\mathrm{ChR} 2^{+/-}\right)$were then backcrossed with female Rosa26-DT mice, which resulted in 4 lines of mice in the $\mathrm{F}_{1}$ generation: LM (Rosa26-DT ${ }^{\mathrm{Mlc2v-Cre}-/-}$, ChAT.ChR2 ${ }^{-/-}$), Chat-

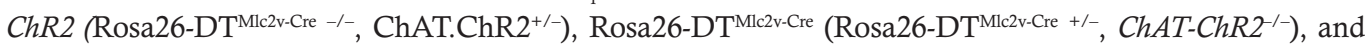

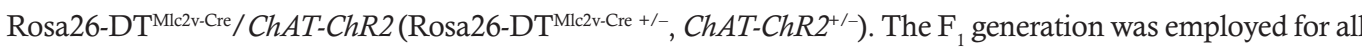


the experiments described herein. Adult mice, age 8-10 weeks, were used for all studies. The mouse colonies were maintained in a pathogen-free environment and were fed pellet food and water ad libitum in accordance with NIH guidelines. Experiments were performed according to approved animal protocols from the Institutional Animal Care and Use Committee at Washington University School of Medicine.

\section{Cardiomyocyte ablation}

To induce cardiomyocyte cell death, mice were injected with DT (2.5 $\mu \mathrm{g} / \mathrm{kg}$, intraperitoneally). In preliminary studies, we determined that the response to a single injection of DT resulted in variability in the amount of tissue injury, whereas the administration of DT on 2 consecutive days resulted in a more consistent tissue injury. Accordingly, for the studies described herein, cardiomyocyte ablation was achieved by injecting DT on 2 consecutive days (day 0 and day 1). LMs were also injected with DT on day 0 and day 1. The animals were sacrificed on day 3 and day 5 after DT injection.

\section{Treatment with pyridostigmine}

Mice were treated with diluent or PYR for 7 days prior to DT injection using an osmotic minipump and then for another 3-5 days after DT injection. Briefly, ALZET mini-pumps (model 1002) were filled with endotoxin-free phosphate PBS (MilliporeSigma) or PYR (MilliporeSigma, $3 \mathrm{mg} / \mathrm{kg} / \mathrm{d}$; ref. 19). The pumps were implanted subcutaneously 1 week prior to DT injection. Surgery was performed according to the guidelines issued by the Animal Studies Committee Policy of Washington University School of Medicine.

\section{Assessment of myocardial injury after DT injection}

We used Evans Blue dye (MilliporeSigma) uptake and serum troponin I levels to assess the degree of tissue injury following DT injection. Evans blue dye uptake was assessed on day 3 after DT injection, as described previously (37). Hearts were examined at the level of the papillary muscle $(\times 20$ magnification) using ImageJ software (NIH). Data are expressed as the percentage area of the myocardium with red fluorescence. Serum troponin I levels were measured by mandibular bleeding on day 3 and 5 after DT injection (38). Troponin levels $<0.03$ were considered nondetectable.

Gravimetric and histological analysis. Mice were euthanized 3-5 days after DT injection, and the hearts were removed and weighed to determine the heart-weight-to-body-weight ratio. Hearts were processed, paraffin-embedded, and stained with hematoxylin and eosin as described previously (39). To evaluate myocyte size, cardiac-fixed frozen sections $(10 \mu \mathrm{m})$ were stained with fluorescent rhodamine-conjugated wheat germ agglutinin at $5 \mu \mathrm{g} / \mathrm{mL}$ in $1 \%$ BSA, $1 \times$ TBS (Vector Laboratories). Fluorescence was visualized using a Zeiss confocal microscope (LSM 700), and digital images were analyzed with Axiovision software (Zeiss), as previously described (39).

\section{RNA extraction, microarray analysis, and RT-qPCR}

Transcriptional profiling of hearts. We performed transcriptional profiling of hearts ( $n=6 /$ condition) from LM mice treated with diluent (LM/PBS) or PYR (LM/PYR) as well as DT-injected Rosa26-DT ${ }^{\text {Mlc2v-Cre }}$ mice treat-

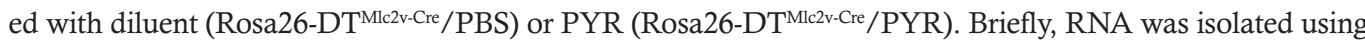
TRIzol (Invitrogen) according to the manufacturer's suggestions. The Dynabead mRNA Purification System (Invitrogen) was used to generate RNAseq libraries as previously described (40). RNAseq was performed using an Illumina HiSeq3000 machine (single end, 50 reads) at the Washington University Genome Technology Access Center. Sequence alignment was obtained through Tophat software (41). Gene-level quantification was performed using HTSeq (version 0.5.1; ref. 42), in which we obtained an average of $1.3 \times 10^{7}$ total feature-assigned reads per sample. The transcripts were filtered $(0.5 \mathrm{cpm}$ and present in at least $50 \%$ of the samples/group), yielding a total of 15,140 transcripts that were classified as detectable. The data are available on the NCBI GEO repository, accession GSE116988. Following normalization for library depth (43), a principal component analysis was performed using Partek Genomics Suite 7.0. The limma-voom procedure was used to identify mRNAs with differential expression between experimental conditions (44). We generated lists of genes that were upregulated following DT injury and normalized by PYR treatment by selecting genes whose expression levels were significantly (fold change $\geq 1.5$ and FDR $<0.05$ ) altered following DT injury in Rosa26-DT ${ }^{\mathrm{Mlc} 2 \mathrm{v}-\mathrm{Cre}} / \mathrm{PBS}$ hearts compared with LM/PBS hearts and that were normalized in DT-injected Rosa26-DT ${ }^{\text {Mlc2v-Cre }}$ mice treated with PYR compared with DT-injected Rosa26-DT ${ }^{\text {Mlc2v-Cre }}$ mice treated with diluent. KEGG pathway analysis was performed on mRNAs with greater than 1.5 -fold change in expres- 
sion level between experimental groups using the appropriate functional annotation module within the 2017 release of the NIH online resource DAVID (https://david.ncifcrf.gov/summary.jsp). Enriched pathways were accepted at FDR $<0.05$ (Benjamini-Hochberg correction procedure). For each pathway, we determined the number of genes that were either downregulated or upregulated in Rosa26-DT ${ }^{\text {Mlc2v-Cre }} / \mathrm{PBS}$ hearts versus LM/PBS hearts and Rosa26-DT ${ }^{\text {Mlc2v-Cre } / P Y R ~ v e r s u s ~ R o s a 26-D T ~}{ }^{\text {Mlc2v-Cre }} /$ PBS hearts. Heatmaps were generated for genes in the most significantly upregulated top 4 KEGG pathways.

Real-time quantitative PCR. Total RNA was isolated from flash-frozen mouse hearts. The ventricles were homogenized with magnetic beads in $1 \mathrm{~mL}$ of TRIzol (Invitrogen). The PureLink RNA Mini kit (Invitrogen) was used to perform the RNA extraction. cDNA was synthesized using the SuperScript III First-Strand Synthesis System from Invitrogen. RT-PCR (real-time PCR) was performed in a QuantStudio3 Real-Time PCR machine (Thermo Fisher Scientific). Quantitative real-time PCR was performed using commercially available predesigned primers for SYBR green-based reactions (Cd68, Ccr2, Tnf, Il6, Il1- $\beta, C c l 2, C c l 7$, and Rplp0 primers were obtained from Integrated DNA Technologies) or TaqMan-based detections (VAChT, Applied Biosystems, see Supplemental Table 1 for details). Relative quantification was performed using expression levels of $R p l p 0$ as a reference.

\section{Assessment of myocardial inflammation}

Myocardial inflammation was assessed by quantifying infiltrating leukocyte subsets using immunofluorescence staining, hematoxylin and eosin staining, and flow cytometry (FACS).

Immunofluorescence. Hearts were fixed in 4\% paraformaldehyde for 48 hours and then transferred to $30 \%$ sucrose for 24 hours prior to embedding in OCT and flash freezing. Prior to immunostaining, frozen sections were washed briefly in PBS and then blocked in 3\% BSA/5\% horse serum. Sections were stained with primary antibodies (see Supplemental Table 2 for details) to CD68 (macrophages) or Ly6G (neutrophils) followed by Alexa Fluor 555-conjugated secondary antibody incubation (A21434, Thermo Fisher Scientific) or with a FITC-conjugated antibody to CD3 (T cells). Fluorescence was visualized via $\times 10$ tile scanning of the entire heart at the midpapillary level using a Zeiss confocal microscope (LSM 700). Images ( 70-130 per section) were stitched together using the ZEN software from Zeiss to form whole midpapillary section images, as described previously (see Supplemental Figure 9 and ref. 39). The extent of inflammation was measured on day 3 by enumerating the total number of $\mathrm{CD}^{+} 8^{+} \mathrm{DAPI}^{+}$cells within a midpapillary myocardial slice, using ImageJ software (NIH). Because the extent of inflammation on day 5 precluded counting of individual $\mathrm{CD}^{+} 8^{+} \mathrm{DAPI}^{+}$cells, we used ImageJ to measure fluorescent intensity (above background) within a midpapillary myocardial slice and then divided the area of fluorescent intensity by the total area of the myocardial slice. Data are expressed as the percentage of inflammatory infiltrate within the myocardium.

Representative pictures were taken at $\times 10$ or $\times 20$ magnification.

Hematoxylin and eosin staining and myocardial inflammatory score. Myocardial inflammation was assessed in myocardial sections that had been stained with hematoxylin and eosin. The degree of myocardial inflammation was scored semiquantitatively in the following manner: 0 , no infiltrate; $1+$, infiltrates involving $<25 \%$ myocardium; $2+$, infiltrates involving $25 \%-50 \%$; $3+$, infiltrates involving $50 \%-75 \%$ of the myocardium; and $4+$, infiltrates involving $75 \%-100 \%$ of the myocardium, as described previously (17).

ELISA. Cardiac tissue lysates were obtained from diluent- or PYR-treated LMs and Rosa26-DT ${ }^{\mathrm{Mlc} 2 \mathrm{vCre}}$ mice. Briefly, cardiac tissue was homogenized in tissue lysate buffer: 0.5\% IGEPAL (MilliporeSigma), $0.5 \%$ sodium deoxycholate (Calbiochem), $0.1 \%$ sodium dodecyl sulfate (MilliporeSigma), $50 \mathrm{mM}$ Tris$\mathrm{HCl}$ (MilliporeSigma), and $150 \mathrm{mM} \mathrm{NaCl}$ (MilliporeSigma), pH 8.00. Protease and phosphatase inhibitors were used to prevent degradation of proteins. A TissueLyser LT (Qiagen) and Stainless Steel Beads (Qiagen) were used to homogenize the samples at 50 oscillations/s (5-10 minutes). Protein levels of CCL2 (Mcp-1) and CCL7 (Mcp-3) were determined by ELISA, using a murine Mcp-1 ELISA (BD OptEIA) and a Mcp-3 murine instant ELISA (Thermo Fisher Scientific), respectively. Final values were expressed as pg/ mg of cardiac tissue. Cardiac extracts from LM mice did not show detectable levels of CCL2. For statistical purposes, a value of 0 was assigned to samples that were not detectable.

Flow cytometry. Cardiac tissue was digested as previously described (15). Cells were resuspended and stained with antibodies (see Supplemental Table 3) for 40-60 minutes at $4^{\circ} \mathrm{C}$ and washed twice with FACS buffer prior to analysis. Compensation controls were generated using single color control samples from stained splenocytes or UltraComp ebeads (Invitrogen). Data were acquired using BD LSR II, BD LSRFortessa, and BD LSRFortessa X-20 analyzers at the Washington University Department of Pathology 
Flow Cytometry and Sorting Core. The gating strategy used has been previously described (see Supplemental Figure 10 and refs. 15, 38). The data obtained by FACS were obtained from a minimum of 3 independent experiments, and the group data are expressed as number of cells/mg of tissue.

Isolation of leukocytes from the blood and spleen. Blood obtained by mandibular bleeding was collected into tubes containing $100 \mathrm{mM}$ EDTA solution, red blood cells were lysed using the PharmLyse $1 \times(\mathrm{BD}$ Pharmingen), and the remaining cells were resuspended in FACS buffer. For analysis of blood, 100,000 events were collected in the cytometer per sample. Data are shown as the number of Ly6C $\mathrm{C}^{\text {hi }}$ cells per 100,000 events. Spleens were removed and minced using $40-\mu \mathrm{m}$ filters in Hank's buffered saline solution as described previously (45). Red blood cells were lysed using Ammonium-Chloride-Potassium Lysis Buffer (Fisher Life Technologies), and the remaining cells were resuspended in FACS buffer. Staining, compensation controls, and acquisition were as described above. T cells, B cells, and neutrophils in the blood and spleen were identified using CD3, CD19, and Ly6G antibodies (for details, see Supplemental Table 3).

Cell sorting and $q R T-P C R$. Sorting of $\mathrm{CD}_{4} 5^{+} \mathrm{Ly} 6 \mathrm{G}^{-} \mathrm{CD} 64^{+} \mathrm{CCR} 2^{+} \mathrm{MHC}-\mathrm{II}^{\mathrm{hi}}$ and $\mathrm{CD} 45^{+} \mathrm{Ly} 6 \mathrm{G}^{-} \mathrm{CD} 64^{+} \mathrm{C}-$ $\mathrm{CR} 2^{+} \mathrm{MHC}^{-\mathrm{II}^{\mathrm{lo}}}$ cells from the hearts of Rosa26-DT ${ }^{\mathrm{Mlc2v-Cre}} / \mathrm{PBS}$ or Rosa26-DT ${ }^{\mathrm{Mlc} 2 \mathrm{~V}-\mathrm{Cre}} / \mathrm{PBS}$ mice (day 3 after DT injection) was performed as described previously (15), using a FACS Aria flow cytometer (BD) at the Washington University Flow Cytometry and Sorting Core. Briefly, CCR2 ${ }^{+} \mathrm{MHC}-\mathrm{II}^{\mathrm{hi}}$ and CCR2 $2^{+} \mathrm{MHC}-\mathrm{II}^{\mathrm{lo}}$ cells were collected in lysis buffer, and RNA was extracted using the Zymo Research QuickRNA MicroPrep (Zymo Research), according to the manufacturer's specifications. RNA was eluted in $11 \mu \mathrm{L}$ RNAse/ DNAse-free water, and $10 \mu \mathrm{L}$ was used for cDNA synthesis (Maxima First Strand cDNA Synthesis kit for RT-PCR, Thermo Fisher Scientific). Quantitative reverse transcription-PCR (RT-PCR) was performed in a QuantStudio3 Real-Time PCR machine (Thermo Fisher Scientific). TaqMan probes for $C c l 2$ and $C c l 7$ were used (for details, see Supplemental Table 1). Gapdh was used as a normalization control.

\section{ipMACs}

Macrophages (referred to as ipMACs) were harvested from the peritoneal cavity of thioglycolate-stimulated C57BL6/J mice, as described previously (38). Cultures of ipMACs were preincubated for 30 minutes with diluent or increasing concentrations $\left(10^{-6} \mu \mathrm{M}\right.$ to $\left.10^{2} \mu \mathrm{M}\right)$ of ACh (MilliporeSigma), muscarine (Tocris) or nicotine (Tocris), prior to stimulation with $100 \mathrm{ng} / \mathrm{mL}$ LPS (LPS-EB Ultrapure; InvivoGen). PYR $(1 \mathrm{mM})$ was added to the cultures of ACh-treated ipMACs in order to prevent ACh degradation by cholinesterase. Drugs were diluted in endotoxin-free PBS for all experiments. The supernatants from the ipMAC cultures were collected after 7 hours and 30 minutes following LPS stimulation, and the levels of secreted CCL2 (MCP-1) and TNF were determined by ELISA, using a commercially available murine Mcp-1 Kit (BD OptEIA) and TNF kit (BD OptEIA). Data ( $n=$ at least 3 independent experiments) are expressed as a percentage of the maximum release of CCL2 or TNF in LPS-treated ipMACs. mACh receptors are G protein-coupled receptors that can be classified into 5 subtypes, M1-M5. Although mACh receptors are activated by the same ACh ligand, the distribution and role of $\mathrm{mACh}$ receptors is cell-type dependent (46). Plated ipMACs were stained with either M1 or M3 primary antibodies (see Supplemental Table 2 for details) followed by staining with Alexa Fluor 488-conjugated secondary antibody (A11034, Thermo Fisher Scientific). DAPI was used to stain the nucleus, and images were acquired in a Zeiss confocal microscope (LSM 700). To investigate the role of M1 and M3 ACh receptors, we preincubated cultures of LPS-stimulated ipMACs with $1 \mu \mathrm{M}$ Telenzepine ( $1 \mu \mathrm{M}$, Tocris), a selective M1 inhibitor, or $1 \mu \mathrm{M}$ 4-DAMP (1,1-dimethyl-4-diphenylacetoxypiperidinium iodide; Tocris), a selective inhibitor of the M3 receptor, for 20 minutes before treatment with $0.1 \mu \mathrm{M}$ muscarine.

\section{Statistics}

All results are presented as mean \pm SEM. The Kaplan-Meier cumulative survival curves were analyzed using the Gehan-Breslo-Wilcoxon test. Statistical comparisons were performed using 2-tailed Student's $t$ test when 2 groups were analyzed or 1-way ANOVA followed by Tukey post hoc testing, where appropriate. Statistical analyses of LPS-stimulated ipMACs treated with different concentrations of ACh, muscarine, and nicotine were performed using 1-way or 2-way ANOVA using generalized linear models procedure with the option ADJUST = TUKEY to account for any missing values. Data were analyzed with GraphPad Prism version 7.04 or SAS version 9.4 in Windows version 10. Results were considered statistically significant when they passed a threshold of $P<0.05$. 


\section{Study approval}

All studies were performed with the approval of the Institutional Animal Care and Use Committee at Washington University School of Medicine, and they follow the guidelines described in the NIH Guide for the Care and Use of Laboratory Animals (National Academies Press, 2011).

\section{Author contributions}

CRR designed and performed experiments and wrote the manuscript. DLM designed experiments and wrote the manuscript. PMB, JS, KJL, LA, and GB designed experiments. SJM analyzed the output of whole-genome RNAseq analyses. CW performed the closed-chest ischemia/reperfusion and sham procedures. All authors edited and approved the final version of the manuscript.

\section{Acknowledgments}

This study was supported by research funds from the NIH (R01 HL-58081, HL-73017-0, HL-089543-01), institutional funds from the Center for Cardiovascular Research at Washington University School of Medicine, and an S10 Shared Instrumentation Grant from the NIH. The authors would like to acknowledge the Washington University Digestive Disease Research Core Center Morphology Core (National Institute of Diabetes and Digestive and Kidney Diseases P30 DK052574) for preparing the histology specimens and to acknowledge the Washington University Flow Cytometry \& Fluorescence Activated Cell Sorting Core for assistance with FACS analysis and cell sorting. We also thank Marco Prado for his helpful advice and for providing the Chat-ChR2 mice.

Address correspondence to: Douglas L. Mann, Division of Cardiology, 660 S. Euclid Avenue, Campus Box 8086, St. Louis, Missouri 63110, USA. Phone: 314.362.8908; Email: dmann@wustl.edu.

1. Steinman L. Elaborate interactions between the immune and nervous systems. Nat Immunol. 2004;5(6):575-581.

2. Evans SS, Repasky EA, Fisher DT. Fever and the thermal regulation of immunity: the immune system feels the heat. Nat Rev Immunol. 2015;15(6):335-349.

3. Kenney MJ, Ganta CK. Autonomic nervous system and immune system interactions. Compr Physiol. 2014;4(3):1177-1200.

4. Borovikova LV, et al. Vagus nerve stimulation attenuates the systemic inflammatory response to endotoxin. Nature. 2000;405(6785):458-462.

5. Wang $\mathrm{H}$, et al. Nicotinic acetylcholine receptor alpha7 subunit is an essential regulator of inflammation. Nature 2003;421(6921):384-388.

6. Olofsson PS, Rosas-Ballina M, Levine YA, Tracey KJ. Rethinking inflammation: neural circuits in the regulation of immunity. Immunol Rev. 2012;248(1):188-204.

7. Roy A, Guatimosim S, Prado VF, Gros R, Prado MA. Cholinergic activity as a new target in diseases of the heart. Mol Med. 2015;20:527-537.

8. Wessler I, Kilbinger H, Bittinger F, Unger R, Kirkpatrick CJ. The non-neuronal cholinergic system in humans: expression, function and pathophysiology. Life Sci. 2003;72(18-19):2055-2061.

9. Roy A, et al. Cardiomyocyte-secreted acetylcholine is required for maintenance of homeostasis in the heart. FASEB J. 2013;27(12):5072-5082.

10. Kakinuma Y, Akiyama T, Okazaki K, Arikawa M, Noguchi T, Sato T. A non-neuronal cardiac cholinergic system plays a protective role in myocardium salvage during ischemic insults. PLoS One. 2012;7(11):e50761.

11. Rocha-Resende C, et al. Non-neuronal cholinergic machinery present in cardiomyocytes offsets hypertrophic signals. J Mol Cell Cardiol. 2012;53(2):206-216.

12. Roy A, et al. Cardiac acetylcholine inhibits ventricular remodeling and dysfunction under pathologic conditions. $F A S E B J$. 2016;30(2):688-701

13. Reardon C, et al. Lymphocyte-derived ACh regulates local innate but not adaptive immunity. Proc Natl Acad Sci USA. 2013;110(4):1410-1415.

14. Kirkpatrick CJ, Bittinger F, Nozadze K, Wessler I. Expression and function of the non-neuronal cholinergic system in endothelial cells. Life Sci. 2003;72(18-19):2111-2116.

15. Lavine KJ, et al. Distinct macrophage lineages contribute to disparate patterns of cardiac recovery and remodeling in the neonatal and adult heart. Proc Natl Acad Sci USA. 2014;111(45):16029-16034.

16. Hogue CW, Stein PK, Apostolidou I, Lappas DG, Kleiger RE. Alterations in temporal patterns of heart rate variability after coronary artery bypass graft surgery. Anesthesiology. 1994;81(6):1356-1364.

17. Kanda T, et al. Modification of viral myocarditis in mice by interleukin-6. Circ Res. 1996;78(5):848-856.

18. Bajpai G, et al. Tissue Resident CCR2- and CCR2+ Cardiac Macrophages Differentially Orchestrate Monocyte Recruitment and Fate Specification Following Myocardial Injury. Circ Res. 2019;124(2):263-278.

19. Gavioli M, et al. Cholinergic signaling exerts protective effects in models of sympathetic hyperactivity-induced cardiac dysfunction. PLoS One. 2014;9(7):e100179.

20. Vaseghi M, et al. Parasympathetic dysfunction and antiarrhythmic effect of vagal nerve stimulation following myocardial infarction. JCI Insight. 2017;2(16):86715. 
21. Wessler I, Kirkpatrick CJ, Racké K. The cholinergic 'pitfall': acetylcholine, a universal cell molecule in biological systems, including humans. Clin Exp Pharmacol Physiol. 1999;26(3):198-205.

22. Kakinuma Y. Future perspectives of a cardiac non-neuronal acetylcholine system targeting cardiovascular diseases as an adjunctive tool for metabolic intervention. Int Immunopharmacol. 2015;29(1):185-188.

23. Sabbah HN, Ilsar I, Zaretsky A, Rastogi S, Wang M, Gupta RC. Vagus nerve stimulation in experimental heart failure. Heart Fail Rev. 2011;16(2):171-178.

24. Byku M, Mann DL. Neuromodulation of the failing heart: Lost in translation? JACC Basic Transl Sci. 2016;1(3):95-106.

25. LaCroix C, Freeling J, Giles A, Wess J, Li YF. Deficiency of M2 muscarinic acetylcholine receptors increases susceptibility of ventricular function to chronic adrenergic stress. Am J Physiol Heart Circ Physiol. 2008;294(2):H810-H820.

26. Tracey KJ. The inflammatory reflex. Nature. 2002;420(6917):853-859.

27. Schwartz PJ, Pagani M, Lombardi F, Malliani A, Brown AM. A cardiocardiac sympathovagal reflex in the cat. Circ Res. 1973;32(2):215-220.

28. Bellinger DL, Lorton D. Autonomic regulation of cellular immune function. Auton Neurosci. 2014;182:15-41.

29. Kakinuma Y, Akiyama T, Sato T. Cholinoceptive and cholinergic properties of cardiomyocytes involving an amplification mechanism for vagal efferent effects in sparsely innervated ventricular myocardium. FEBS J. 2009;276(18):5111-5125

30. Rocha JA, et al. Increase in cholinergic modulation with pyridostigmine induces anti-inflammatory cell recruitment soon after acute myocardial infarction in rats. Am J Physiol Regul Integr Comp Physiol. 2016;310(8):R697-R706.

31. Nordström P, Religa D, Wimo A, Winblad B, Eriksdotter M. The use of cholinesterase inhibitors and the risk of myocardial infarction and death: a nationwide cohort study in subjects with Alzheimer's disease. Eur Heart J. 2013;34(33):2585-2591.

32. Kawano H, Okada R, Yano K. Histological study on the distribution of autonomic nerves in the human heart. Heart Vessels. 2003;18(1):32-39.

33. Zhang W, et al. Necrotic myocardial cells release damage-associated molecular patterns that provoke fibroblast activation in vitro and trigger myocardial inflammation and fibrosis in vivo. $J$ Am Heart Assoc. 2015;4(6):e001993.

34. Sundman E, Olofsson PS. Neural control of the immune system. Adv Physiol Educ. 2014;38(2):135-139.

35. Zhao S, et al. Cell type-specific channelrhodopsin-2 transgenic mice for optogenetic dissection of neural circuitry function. Nat Methods. 2011;8(9):745-752.

36. Kolisnyk B, et al. ChAT-ChR2-EYFP mice have enhanced motor endurance but show deficits in attention and several additional cognitive domains. J Neurosci. 2013;33(25):10427-10438.

37. Tzeng HP, et al. Dysferlin mediates the cytoprotective effects of TRAF2 following myocardial ischemia reperfusion injury. $J A m$ Heart Assoc. 2014;3(1):e000662.

38. Adamo L, et al. Modulation of subsets of cardiac B lymphocytes improves cardiac function after acute injury. JCI Insight. 2018;3(11):120137.

39. Evans S, et al. TNF receptor-activated factor 2 mediates cardiac protection through noncanonical NF- $\mathrm{B}$ signaling. JCI Insight. 2018;3(3):98278.

40. Matkovich SJ, Dorn GW. Deep sequencing of cardiac microRNA-mRNA interactomes in clinical and experimental cardiomyopathy. Methods Mol Biol. 2015;1299:27-49.

41. Trapnell C, Pachter L, Salzberg SL. TopHat: discovering splice junctions with RNA-Seq. Bioinformatics. 2009;25(9):1105-1111.

42. Anders S, Pyl PT, Huber W. HTSeq-a Python framework to work with high-throughput sequencing data. Bioinformatics. 2015;31(2):166-169.

43. Anders S, Huber W. Differential expression analysis for sequence count data. Genome Biol. 2010;11(10):R106.

44. Zhou X, Lindsay H, Robinson MD. Robustly detecting differential expression in RNA sequencing data using observation weights. Nucleic Acids Res. 2014;42(11):e91.

45. Epelman S, Lavine KJ, Randolph GJ. Origin and functions of tissue macrophages. Immunity. 2014;41(1):21-35.

46. Saternos HC, et al. Distribution and function of the muscarinic receptor subtypes in the cardiovascular system. Physiol Genomics. 2018;50(1):1-9 